QUARTERLY OF APPLIED MATHEMATICS

VOLUME LXIII, NUMBER 2

JUNE 2005, PAGES 369-400

S $0033-569 \mathrm{X}(05) 00963-3$

Article electronically published on May 4, 2005

\title{
ASYMPTOTIC BEHAVIOUR OF PRESSURE AND STRESSES IN A THIN FILM FLOW WITH A ROUGH BOUNDARY
}

\author{
BY \\ NADIA BENHABOUCHA (CNRS UMR 5585, Maply, Bat J. Braconnier, Univ. Lyon1, 69622 \\ Villeurbanne Cedex France), \\ MICHÈLE CHAMBAT (CNRS UMR 5585, Maply, Bat J. Braconnier, Univ. Lyon1, 69622 \\ Villeurbanne Cedex France), \\ AND \\ IONEL CIUPERCA (CNRS UMR 5585, Maply, Bat J. Braconnier, Univ. Lyon1, 69622 \\ Villeurbanne Cedex France )
}

\begin{abstract}
We study the asymptotic behaviour of an incompressible viscous flow in a narrow gap with a thickness of order $\eta$ and a rough surface. The roughness is defined by a quasi-periodical function with period $\epsilon$. In both cases, when $\eta$ is smaller or at the same order as $\epsilon$, we obtain different Reynolds equations for the pressure. We have also studied the convergence of the stresses on the rough boundary and we discuss the different cases.
\end{abstract}

1. Introduction. In this paper, we study the asymptotic behaviour of an incompressible fluid in a narrow gap with a thickness of order $\eta$, the boundaries being rough with a roughness period $\epsilon$. Neglecting the inertial terms and the exterior forces, we consider the following Stokes system:

$$
\left(\mathcal{P}^{\eta \epsilon}\right) \quad\left\{\begin{array}{l}
\mu \triangle u^{\eta \epsilon}=\nabla p^{\eta \epsilon} \\
\operatorname{div} u^{\eta \epsilon}=0
\end{array}\right.
$$

with adhering boundary conditions, where $u^{\eta \epsilon}, p^{\eta \epsilon}$ are the velocity and the pressure and $\mu$ is the viscosity of the fluid.

In some papers [1, 4, 17], the roughness of the boundary is taken into account by means of a periodic function, with a small height amplitude of the same order of magnitude as the period, while the flow height is not small. In [14, 13, studies appear related to lubrication from a mechanical point of view. Mathematical asymptotic studies using two parameters $\eta$ and $\epsilon$, which are supposed to tend to zero, appear in a formal way in [6]

Received January 3, 2005.

2000 Mathematics Subject Classification. Primary 76D07, 76D08, 78M35 78M40.

Key words and phrases. Homogenization, rough boundary, Reynolds equation, asymptotic stresses.

E-mail address: Nadia.Benhaboucha@maply.univ-lyon1.fr

E-mail address: chambat@maply.univ-lyon1.fr

E-mail address: ciuperca@maply.univ-lyon1.fr 
and rigorously in [5]. The authors proved that the way the two parameters tend to zero is primordial, since the limiting equations are different whether $\epsilon$ tends to zero faster, slower, or at the same rate as $\eta$. It is a very common situation when problems with several small parameters lead to different limit models [2, 8, 10, 18. In [5] and [6] the authors considered the case

$$
\eta=\lambda \epsilon \quad \text { with } \lambda>0
$$

and they obtained a limit problem $\left(\mathcal{P}^{\lambda}\right)$ under the hypothesis that the gap is uniformly periodic.

In the present study, we consider a quasi-periodic gap. The significant variables for mechanicians are the pressure and the forces exerted by the fluid on the surface walls. We are interested in the asymptotic study of both the pressure and the boundary stresses, so we set:

$$
\eta=\lambda \epsilon^{\alpha} \quad \text { with } \quad \lambda>0, \quad \alpha \geq 1
$$

the case $0<\alpha<1$, being meaningless for the lubrication.

The case $\alpha=1$ corresponds to a narrow gap of the same order of magnitude as the roughness period and generalizes the results in [5] for the pressure. The asymptotic pressure satisfies a homogenized Reynolds equation $\left(\mathcal{P}^{\lambda}\right)$ whose coefficients depend on solutions to local problems being 3D Stokes equations.

The case $\alpha>1$ corresponds to a narrow gap smaller than the roughness period. The asymptotic pressure satisfies a homogenized Reynolds equation $\left(\mathcal{P}^{\star}\right)$ whose coefficients depend on solutions to local problems being $2 \mathrm{D}$ elliptic equations. The same limit problem $\left(\mathcal{P}^{\star}\right)$ is obtained by passing to the limit $\lambda \rightarrow 0$ in $\left(\mathcal{P}^{\lambda}\right)$. On the other hand, by passing to the limit $\eta \rightarrow 0$ and then $\epsilon \rightarrow 0$ in $\left(\mathcal{P}^{\eta \epsilon}\right)$, the same limit problem $\left(\mathcal{P}^{\star}\right)$ is also obtained (see [5]).

The main contribution of this paper concerns the stress vector on the rough boundary for which the situation is more complex. A priori estimates lead to another critical exponent $\alpha=3 / 2$. For $\alpha>3 / 2$, the stress limit behaviour is the same as letting first $\eta$ then $\epsilon$ tend to zero in $\left(\mathcal{P}^{\eta \epsilon}\right)$. For $\alpha=3 / 2$, a supplementary term depending on the parameter $\lambda$ appears in the limit. For $1<\alpha<3 / 2$, the two first components of the stresses are not bounded; nevertheless, we characterise their behaviour by obtaining the convergences after multiplication by $\epsilon^{3-2 \alpha}$.

The paper is organized as follows. The problem is presented in Section 2 with two equivalent variational formulations, the first after rescaling and the second after rescaling and flattening the real domain. The main results are given in Section 3 and concern the convergence of the pressure and that of the stresses. The proofs of each previous result are given in Section 4 for the pressure and in Section 5 for the stresses.

2. Statement of the problem. Let us begin with some notation. We write $Z=$ $\left(z_{1}, z_{2}, z_{3}\right)$ for a current point in $\mathbb{R}^{3}$ and $z=\left(z_{1}, z_{2}\right)$ for its projection in $\mathbb{R}^{2} . \omega$ is an open set in $\mathbb{R}^{2}$ with a Lipschitz boundary and $Y=[0,1]^{2}$ is the basic cell in $\mathbb{R}^{2}$. We consider 
a smooth function $h=h(z, y)$, such that:

$$
h \in \mathcal{C}^{2}\left(w \times \mathbb{R}^{2}\right), h \text { is periodic in } y, \quad \exists a, b>0 \quad a \leq h(z, y) \leq b \quad \forall z \in w, y \in Y .
$$

We set

$$
h_{\text {min }}=\min _{(z, y) \in \omega \times Y} h(z, y), \quad h_{\max }=\max _{(z, y) \in \omega \times Y} h(z, y)
$$

and

$$
\left.\Omega_{\min }=\omega \times\right] 0, h_{\min }\left[, \quad \Omega_{\max }=\omega \times\right] 0, h_{\max }[.
$$

We introduce two small parameters, $\epsilon$ related to the roughness wavelength and $\eta$ related to the thickness of the gap between the two surfaces, so that the three-dimensional domain occupied by the fluid is

$$
\Omega^{\eta \epsilon}=\left\{\left(z, z_{3}\right) / \quad z \in \omega \text { and } 0<z_{3}<\eta h^{\epsilon}(z)\right\},
$$

where

$$
h^{\epsilon}(z)=h\left(z, \frac{z}{\epsilon}\right) \quad \forall z \in \omega .
$$

In the following, we will need two different scalings.

$\left.\Omega^{\epsilon}=\omega \times\right] 0, h^{\epsilon}\left[\right.$ is the transformation of the domain $\Omega^{\eta \epsilon}$ with rescaled thickness

$$
x=z, \text { and } x_{3}=\frac{z_{3}}{\eta} ;
$$

$\Omega=\omega \times] 0,1\left[\right.$ is the transformation of the domain $\Omega^{\eta \epsilon}$ by the change of variables

$$
t=z \text {, and } t_{3}=\frac{z_{3}}{\eta h^{\epsilon}} .
$$

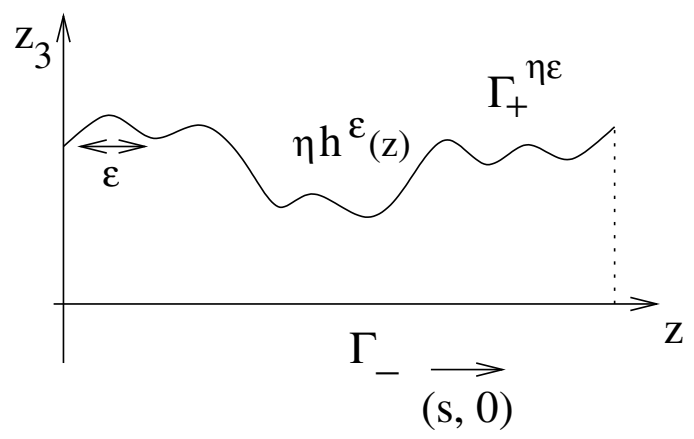

FIG. 1. Domain $\Omega^{\eta \epsilon}$

We will denote, for any function $\varphi$, its average on the basic cell $Y$ by

$$
\widetilde{\varphi}=\int_{Y} \varphi d y
$$

and for any function $\varphi$ defined on $\Omega^{\epsilon}$, we denote by

$$
\bar{\varphi}=\left\{\begin{array}{lll}
\varphi & \text { on } & \Omega^{\epsilon}, \\
0 & \text { on } & \Omega_{\max }-\Omega^{\epsilon}
\end{array}\right.
$$


its extension by zero to $\Omega_{\max }$.

REMARK 2.1. Throughout this paper, for the sake of simplicity, the velocity and pressure will be denoted by $u^{\epsilon}$ and $p^{\epsilon}$ instead of $u^{\eta \epsilon}$ and $p^{\eta \epsilon}$, since $\eta$ and $\epsilon$ are linked by the relation (1.2). Moreover, the same notation will be used for a function defined on the three different domains $\Omega^{\eta \epsilon}, \Omega^{\epsilon}$, and $\Omega$.

Let $s=\left(s_{1}, s_{2}\right)$ be a given vector in $\mathbb{R}^{2}$ and let $g$ be a given function in $\left(H^{\frac{1}{2}}\left(\partial \Omega_{\text {min }}\right)\right)^{3}$, which does not depend on $\eta$ and $\epsilon$, such that

$$
g\left(x, h_{\text {min }}\right)=0 \quad g(x, 0)=\left(s_{1}, s_{2}, 0\right) \quad \forall x \in \omega
$$

and

$$
\int_{\partial \Omega_{\min }} g \cdot n d \sigma=0 .
$$

From the hypotheses (2.5) and (2.6) on $g, G=\left(G_{1}, G_{2}, G_{3}\right)$ in $H^{1}\left(\Omega_{\text {min }}\right)^{3}$ exists such that $\operatorname{div} G=0$ in $\Omega_{\text {min }}$ and $G=g$ on $\partial \Omega_{\min }$ (see [16]). We still denote by $G$ its extension by zero to $\Omega^{\epsilon}$. Let $G^{\epsilon}=\left(G_{1}, G_{2}, \eta G_{3}\right)$, which belongs to $H^{1}\left(\Omega^{\epsilon}\right)$.

The variational formulation of the Stokes problem $\left(\mathcal{P}^{\eta \epsilon}\right)$ in $\Omega^{\epsilon}$ follows:

Find $u^{\epsilon}$ in $H^{1}\left(\Omega^{\epsilon}\right)^{3}, p^{\epsilon}$ in $L_{0}^{2}\left(\Omega^{\epsilon}\right)$ such that

$$
\begin{gathered}
\mu \int_{\Omega^{\epsilon}} \nabla_{\eta} u^{\epsilon} \cdot \nabla_{\eta} \phi d x d x_{3}=\int_{\Omega^{\epsilon}} p^{\epsilon} \operatorname{div}_{\eta} \phi d x d x_{3} \quad \forall \phi \in H_{0}^{1}\left(\Omega^{\epsilon}\right)^{3} \\
\int_{\Omega^{\epsilon}} \operatorname{div}_{\eta} u^{\epsilon} q d x d x_{3}=0 \quad \forall q \in L^{2}\left(\Omega^{\epsilon}\right) \\
u^{\epsilon}-G^{\epsilon} \in\left(H_{0}^{1}\right)\left(\Omega^{\epsilon}\right)^{3}
\end{gathered}
$$

where

$$
\nabla_{\eta}=\left(\frac{\partial}{\partial x_{1}}, \frac{\partial}{\partial x_{2}}, \frac{1}{\eta} \frac{\partial}{\partial x_{3}}\right), \quad \operatorname{div}_{\eta}=\frac{\partial}{\partial x_{1}}+\frac{\partial}{\partial x_{2}}+\frac{1}{\eta} \frac{\partial}{\partial x_{3}} .
$$

The variational formulation of the Stokes problem in $\Omega$ follows:

Find $u^{\epsilon}$ in $H^{1}(\Omega)^{3}, p^{\epsilon}$ in $L^{2}(\Omega)$ with $\int_{\Omega} h^{\epsilon} p^{\epsilon} d x d t_{3}=0$ such that

$$
\begin{aligned}
& \mu \int_{\Omega} \sum_{j=1}^{3}\left[\sum_{i=1}^{2}\left(\frac{\partial}{\partial t_{i}}\left(\eta h^{\epsilon} u_{j}^{\epsilon}\right)+\frac{\partial}{\partial t_{3}}\left(-\eta t_{3} \frac{\partial h^{\epsilon}}{\partial t_{i}} u_{j}^{\epsilon}\right)\right)\right. \\
& \left.\cdot\left(\frac{\partial}{\partial t_{i}}\left(\eta h^{\epsilon} \varphi_{j}\right)+\frac{\partial}{\partial t_{3}}\left(-\eta t_{3} \frac{\partial h^{\epsilon}}{\partial t_{i}} \varphi_{j}\right)\right)+\frac{\partial u_{j}^{\epsilon}}{\partial t_{3}} \frac{\partial \varphi_{j}}{\partial t_{3}}\right] \frac{d t d t_{3}}{\eta h^{\epsilon}} \\
& =\int_{\Omega} p^{\epsilon}\left[\sum_{i=1}^{2}\left(\frac{\partial}{\partial t_{i}}\left(\eta h^{\epsilon} \varphi_{i}\right)+\frac{\partial}{\partial t_{3}}\left(-\eta t_{3} \frac{\partial h^{\epsilon}}{\partial t_{i}} \varphi_{i}\right)\right)+\frac{\partial \varphi_{3}}{\partial t_{3}}\right] d t d t_{3} \quad \forall \varphi \in H_{0}^{1}(\Omega)^{3}
\end{aligned}
$$

$$
\begin{array}{cc}
\operatorname{div}_{t t_{3}}\left(\eta h^{\epsilon} B^{\epsilon} \cdot u^{\epsilon}\right)=0 & \text { in } \Omega \\
u^{\epsilon}-G^{\epsilon} \in\left(H_{0}^{1}\right)(\Omega)^{3} &
\end{array}
$$


where $B^{\epsilon}$ is the Jacobian matrix

$$
B^{\epsilon}=\left(\begin{array}{ccc}
1 & 0 & 0 \\
0 & 1 & 0 \\
\frac{-t_{3}}{h^{\epsilon}} \frac{\partial h^{\epsilon}}{\partial t_{1}} & \frac{-t_{3}}{h^{\epsilon}} \frac{\partial h^{\epsilon}}{\partial t_{2}} & \frac{1}{\eta h^{\epsilon}}
\end{array}\right)
$$

3. Main results. In this section, we only state the convergence results; the proofs are shown in Sections 4 and 5.

3.1. Convergence theorems for the pressure. It is supposed that $\eta=\lambda \epsilon^{\alpha}$ and the convergence results are different whether $\alpha=1$ or $\alpha>1$. So we consider the two different cases.

3.1.1. The case $\alpha=1$. We first define the sets

$$
\begin{gathered}
B_{x}=\left\{\left(y, x_{3}\right), \quad y \in Y, \quad 0<x_{3}<h(x, y)\right\} \\
Q=\bigcup_{x \in \omega}\{x\} \times B_{x}=\left\{\left(x, x_{3}, y\right), \quad x \in \omega, \quad y \in Y, \quad 0<x_{3}<h(x, y)\right\}
\end{gathered}
$$

the operators

$$
\begin{aligned}
\triangle_{\lambda} & =\frac{\partial^{2}}{\partial y_{1}^{2}}+\frac{\partial^{2}}{\partial y_{2}^{2}}+\frac{1}{\lambda^{2}} \frac{\partial^{2}}{\partial x_{3}^{2}} \\
\nabla_{\lambda} & =\left(\frac{\partial}{\partial y_{1}}, \frac{\partial}{\partial y_{2}}, \frac{1}{\lambda} \frac{\partial}{\partial x_{3}}\right) \\
\operatorname{div}_{\lambda} & =\frac{\partial}{\partial y_{1}}+\frac{\partial}{\partial y_{2}}+\frac{1}{\lambda} \frac{\partial}{\partial x_{3}}
\end{aligned}
$$

and the spaces

$$
\begin{aligned}
& C_{0 \#}^{\infty}\left(B_{x}\right)=\left\{\varphi \in C^{\infty}\left(B_{x}\right), \quad \varphi \text { is } y \text { periodic, } \varphi(y, 0)=\varphi(y, h(x, y))=0\right\} \\
& H_{\#}^{1}\left(B_{x}\right)=\left\{\varphi \in H^{1}\left(B_{x}\right), \varphi \text { is } y \text { periodic }\right\} \\
& H_{0 \#}^{1}\left(B_{x}\right)=\left\{\phi \in H_{\#}^{1}\left(B_{x}\right), \varphi(y, 0)=\varphi(y, h(x, y))=0\right\} .
\end{aligned}
$$

We also introduce the following local problems:

Find $\left(w^{k \lambda}, \pi^{k \lambda}\right)(k=1,2)$ in $\left(H_{0 \#}^{1}\left(B_{x}\right)\right)^{3} \times L_{0}^{2}\left(B_{x}\right)$ such that

$$
\left\{\begin{array}{l}
\mu \int_{B_{x}} \nabla_{\lambda} w^{k \lambda} \cdot \nabla_{\lambda} \varphi d y d x_{3}=\int_{B_{x}} \pi^{k \lambda} \operatorname{div}_{\lambda} \varphi d y d x_{3}-\int_{B_{x}} \varphi_{k} d y d x_{3} \quad \forall \varphi \in\left(H_{0 \#}^{1}\left(B_{x}\right)\right)^{3} \\
\operatorname{div}_{\lambda} w^{k \lambda}=0 .
\end{array}\right.
$$


Find $\left(w^{3 \lambda}, \pi^{3 \lambda}\right)$ in $H_{\#}^{1}\left(B_{x}\right) \times L_{0}^{2}\left(B_{x}\right)$ such that

$$
\left\{\begin{array}{l}
\mu \int_{B_{x}} \nabla_{\lambda} w^{3 \lambda} \cdot \nabla_{\lambda} \varphi d y d x_{3}=\int_{B_{x}} \pi^{3 \lambda} \operatorname{div}_{\lambda} \varphi d y d x_{3} \quad \forall \varphi \in\left(H_{0 \#}^{1}\left(B_{x}\right)\right)^{3} \\
\operatorname{div}_{\lambda} w^{3 \lambda}=0 \\
w^{3 \lambda}=\left(s_{1}, s_{2}, 0\right) \text { on } x_{3}=0 \\
w^{3 \lambda}=(0,0,0) \text { on } x_{3}=h(x, y) .
\end{array}\right.
$$

The convergence of the pressure is given by the following theorem.

Theorem 3.1. For $\alpha=1, \eta^{2} p^{\epsilon}$ two-scale converges in $\Omega$ to $p^{\lambda}(x)$, the unique solution in $H^{1}(\omega)$ of

$$
\left\{\begin{array}{l}
\sum_{i, j=1}^{2} \int_{\omega} a_{i j}^{\lambda} \frac{\partial p^{\lambda}}{\partial x_{j}} \frac{\partial \varphi}{\partial x_{i}} d x=\sum_{i=1}^{2} \int_{\omega} b_{i}^{\lambda} \frac{\partial \varphi}{\partial x_{i}}-\int_{\partial \omega} f . n \varphi d \sigma \quad \forall \varphi \in H^{1}(\omega) \\
\int_{\omega} \tilde{h} p^{\lambda}(t) d t=0
\end{array}\right.
$$

with

$$
\begin{gathered}
a_{i j}^{\lambda}(x)=-\int_{B_{x}} w_{i}^{j \lambda} d y d x_{3}, \\
b_{i}^{\lambda}=\int_{B_{x}} w_{i}^{3 \lambda} d y d x_{3} \quad i=1,2, \\
f_{i}=\int_{0}^{h_{\text {min }}} g_{i} \quad i=1,2 d x_{3},
\end{gathered}
$$

and $w^{k \lambda}$ the unique solutions of the local problems given in (3.2) and (3.3).

3.1.2. The case $\alpha>1$. In this case, due to the fact that $\eta$ tends to zero faster than $\epsilon$, the local problems are two-dimensional problems and independent of $\lambda$. Setting

$$
H_{p e r}^{1}(Y)=\left\{\varphi \in H^{1}(Y), \quad \varphi \text { is } y \text {-periodic }\right\},
$$

we define the following local problems:

Find $\pi^{k}(k=1,2)$ in $H_{p e r}^{1}(Y) \cap L_{0}^{2}(Y)$ such that

$$
\int_{Y} h^{3}\left(e_{k}+\nabla_{y} \pi^{k}\right) \cdot \nabla_{y} \phi d y=0 \quad \forall \phi \in H_{p e r}^{1}(Y)
$$

where $\left\{e_{1}, e_{2}\right\}$ is the canonical base in $\mathbb{R}^{2}$.

Find $\pi^{3}$ in $H_{p e r}^{1}(Y) \cap L_{0}^{2}(Y)$ such that

$$
\int_{Y} \frac{h^{3}}{6 \mu} \nabla_{y} \pi^{3} \cdot \nabla_{y} \phi d y=\int_{Y} h s . \nabla_{y} \phi d y \quad \forall \phi \in H_{p e r}^{1}(Y) .
$$

The convergence of the pressure is now given by 
Theorem 3.2. For $\alpha>1, \eta^{2} p^{\epsilon}$ two-scale converges in $\Omega$ to $p(x)$, the unique solution in $H^{1}(\omega)$ of

$$
\left\{\begin{array}{l}
\sum_{i, j=1}^{2} \int_{\omega} a_{i j} \frac{\partial p}{\partial x_{i}} \frac{\partial \varphi}{\partial x_{j}} d x=\int_{w} \sum_{i=1}^{2} b_{i}(x) \frac{\partial \varphi}{\partial x_{i}} d x-\int_{\partial w} f \varphi d x \quad \forall \varphi \in H^{1}(w) \\
\int_{\omega} \widetilde{h} p(x) d x=0
\end{array}\right.
$$

with $f$ as in Theorem 3.1 and

$$
\begin{aligned}
a_{i j}(x) & =\int_{Y} \frac{h^{3}}{12 \mu}\left(\delta_{i j}+\frac{\partial \pi^{i}}{\partial y_{j}}\right) d y \quad i, j=1,2 \\
b_{i}(x) & =\int_{Y}\left(-\frac{h^{3}}{12 \mu} \frac{\partial \pi^{3}}{\partial y_{i}}+\frac{\tilde{h}}{2} s_{i}\right) d y \quad i=1,2 .
\end{aligned}
$$

3.2. Convergence theorems for the stresses. The stress tensor $\sigma^{\epsilon}=\left(\sigma_{i j}^{\epsilon}\right)_{i, j=1,2,3}$ in $\Omega^{\eta \epsilon}$ is defined by

$$
\sigma_{i j}^{\epsilon}=-p^{\epsilon} \delta_{i j}+\mu\left(\frac{\partial u_{i}^{\epsilon}}{\partial z_{j}}+\frac{\partial u_{j}^{\epsilon}}{\partial z_{i}}\right) .
$$

We introduce the normalized stress tensor

$$
\Sigma^{\epsilon}=\left(\begin{array}{ccc}
\eta \sigma_{11}^{\epsilon} & \eta \sigma_{12}^{\epsilon} & \eta \sigma_{13}^{\epsilon} \\
\eta \sigma_{21}^{\epsilon} & \eta \sigma_{22}^{\epsilon} & \eta \sigma_{23}^{\epsilon} \\
\eta^{2} \sigma_{31}^{\epsilon} & \eta^{2} \sigma_{32}^{\epsilon} & \eta^{2} \sigma_{33}^{\epsilon}
\end{array}\right)
$$

and the normalized stresses on the boundary $\partial \Omega^{\eta \epsilon}$, where $n^{\epsilon}$ is the unit normal exterior to $\partial \Omega^{\eta \epsilon}$ :

$$
V^{\epsilon}=\Sigma^{\epsilon} \cdot n^{\epsilon} \text {. }
$$

The only boundary stresses for such a thin domain which have engineering applications are

$$
V_{+}^{\epsilon}=V_{/_{\Gamma_{+}}{ }^{\epsilon}}^{\epsilon} \text { and } V_{-}^{\epsilon}=V_{/ \Gamma_{-}}^{\epsilon} .
$$

Due to the roughness of $\Gamma_{+}^{\eta \epsilon}$, the results are different on the upper and lower surfaces.

3.2.1. Convergence of the stresses on the rough boundary. The boundary $\Gamma_{+}^{\epsilon}$ of the rescaled domain $\Omega^{\epsilon}$ still depends on $\epsilon$. So it is necessary to work in the fixed domain $\Omega$. The stress tensor is written in $\Omega$ by

$$
\begin{gathered}
\sigma_{i i}^{\epsilon}=-p^{\epsilon}+2 \mu\left(\frac{\partial u_{i}^{\epsilon}}{\partial t_{i}}-2 \frac{\partial h^{\epsilon}}{\partial t_{i}} \frac{t_{3}}{h^{\epsilon}} \frac{\partial u_{i}^{\epsilon}}{\partial t_{3}}\right) \quad i=1,2 \\
\sigma_{33}^{\epsilon}=-p^{\epsilon}+\mu \frac{2}{\eta h^{\epsilon}} \frac{\partial u_{3}^{\epsilon}}{\partial t_{3}} \\
\sigma_{i j}^{\epsilon}=\mu\left(\frac{\partial u_{i}^{\epsilon}}{\partial t_{j}}-2 \frac{\partial h^{\epsilon}}{\partial t_{j}} \frac{t_{3}}{h^{\epsilon}} \frac{\partial u_{i}^{\epsilon}}{\partial t_{3}}+\frac{\partial u_{j}^{\epsilon}}{\partial t_{i}}-2 \frac{\partial h^{\epsilon}}{\partial t_{i}} \frac{t_{3}}{h^{\epsilon}} \frac{\partial u_{j}^{\epsilon}}{\partial t_{3}}\right) \quad i \neq j=1,2 \\
\sigma_{i 3}^{\epsilon}=\sigma_{3 j}^{\epsilon}=\mu\left(\frac{1}{\eta h^{\epsilon}} \frac{\partial u_{i}^{\epsilon}}{\partial t_{3}}+\frac{\partial u_{3}^{\epsilon}}{\partial t_{i}}-2 \frac{\partial h^{\epsilon}}{\partial t_{i}} \frac{t_{3}}{h^{\epsilon}} \frac{\partial u_{3}^{\epsilon}}{\partial t_{3}}\right) \quad i=1,2
\end{gathered}
$$


and the stress vector $V_{+}^{\epsilon}$ on the boundary $\Gamma_{+}$, defined by the equation $t_{3}=1$, is

$$
V_{+}^{\epsilon}=\left(\begin{array}{c}
-\eta^{2} \sigma_{11}^{\epsilon} \frac{\partial h^{\epsilon}}{\partial t_{1}}-\eta^{2} \sigma_{12}^{\epsilon} \frac{\partial h^{\epsilon}}{\partial t_{2}}+\eta \sigma_{13}^{\epsilon} \\
-\eta^{2} \sigma_{21}^{\epsilon} \frac{\partial h^{\epsilon}}{\partial t_{1}}-\eta^{2} \sigma_{22}^{\epsilon} \frac{\partial h^{\epsilon}}{\partial t_{2}}+\eta \sigma_{23}^{\epsilon} \\
-\eta^{3} \sigma_{31}^{\epsilon} \frac{\partial h^{\epsilon}}{\partial t_{1}}-\eta^{3} \sigma_{32}^{\epsilon} \frac{\partial h^{\epsilon}}{\partial t_{2}}+\eta^{2} \sigma_{33}^{\epsilon}
\end{array}\right) \cdot \frac{1}{\sqrt{1+\eta^{2}\left(\frac{\partial h^{\epsilon}}{\partial t_{1}}\right)^{2}+\eta^{2}\left(\frac{\partial h^{\epsilon}}{\partial t_{2}}\right)^{2}}} .
$$

As for the pressure, it is necessary to consider different cases. The first convergence result is

Theorem 3.3. For $\alpha=1,\left(\epsilon V_{+1}^{\epsilon}, \epsilon V_{+2}^{\epsilon}, V_{+3}^{\eta \epsilon}\right)$ converges in $\left(H_{00}^{\frac{1}{2}}\left(\Gamma_{+}\right)\right)^{\prime}{ }^{3}$ to

$$
V_{+}^{\lambda}=\left(\begin{array}{c}
p^{\lambda}\left(\int_{Y} \frac{\lambda^{2} \frac{\partial h}{\partial y_{1}}}{\sqrt{1+\lambda^{2}\left(\frac{\partial h}{\partial y_{1}}\right)^{2}+\lambda^{2}\left(\frac{\partial h}{\partial y_{2}}\right)^{2}}} d y\right) \\
p^{\lambda}\left(\int_{Y} \frac{\lambda^{2} \frac{\partial h}{\partial y_{2}}}{\sqrt{1+\lambda^{2}\left(\frac{\partial h}{\partial y_{1}}\right)^{2}+\lambda^{2}\left(\frac{\partial h}{\partial y_{2}}\right)^{2}}} p^{\lambda} d y\right) \\
p^{\lambda}\left(\int_{Y} \frac{-\lambda^{2}}{\sqrt{1+\lambda^{2}\left(\frac{\partial h}{\partial y_{1}}\right)^{2}+\lambda^{2}\left(\frac{\partial h}{\partial y_{2}}\right)^{2}}} d y\right)
\end{array}\right) .
$$

For $\alpha$ greater than 1 , a subcritical case $\alpha=\frac{3}{2}$ appears to lead to three supplementary convergence results.

Theorem 3.4. For $1<\alpha<\frac{3}{2}$, $\left(\epsilon^{3-2 \alpha} V_{+1}^{\epsilon}, \epsilon^{3-2 \alpha} V_{+2}^{\epsilon}, V_{+3}^{\eta \epsilon}\right)$ converges in $H^{-1}(\omega) \times$ $H^{-1}(\omega) \times\left(H_{00}^{\frac{1}{2}}\left(\Gamma_{+}\right)\right)^{\prime}$ to

$$
F_{+}^{\lambda}=\left(\begin{array}{c}
-\frac{\lambda^{2}}{2} p \widetilde{\left(\frac{\partial h}{\partial y_{1}}\right)^{3}} \\
\widetilde{-\frac{\lambda^{2}}{2} p \widetilde{\left(\frac{\partial h}{\partial y_{2}}\right)^{3}}} \\
-p(x)
\end{array}\right)
$$

For $\alpha=\frac{3}{2}, V_{+}^{\epsilon}$ converges in $H^{-1}(\omega) \times H^{-1}(\omega) \times\left(H_{00}^{\frac{1}{2}}\left(\Gamma_{+}\right)\right)^{\prime}$ to

$$
V_{+}^{\lambda}=\left(\begin{array}{c}
-\frac{\lambda^{2}}{2} p \widetilde{\left(\frac{\partial h}{\partial y_{1}}\right)^{3}}+\frac{\partial}{\partial t_{1}}(p \tilde{h})-\sum_{j=1}^{2} \frac{\partial p}{\partial t_{j}} c_{1 j}(t)-d_{1}(t) \\
-\frac{\lambda^{2}}{2} p \widetilde{\left.\frac{\partial h}{\partial y_{2}}\right)^{3}}+\frac{\partial}{\partial t_{2}}(p \tilde{h})-\sum_{j=1}^{2} \frac{\partial p}{\partial t_{j}} c_{2 j}(t)-d_{2}(t) \\
-p(x)
\end{array}\right) .
$$


For $\alpha>\frac{3}{2}, V_{+}^{\epsilon}$ converges in $H^{-1}(\omega) \times H^{-1}(\omega) \times\left(H_{00}^{\frac{1}{2}}\left(\Gamma_{+}\right)\right)^{\prime}$ to

$$
V_{+}=\left(\begin{array}{c}
\frac{\partial}{\partial t_{1}}(p \tilde{h})-\sum_{j=1}^{2} \frac{\partial p}{\partial t_{j}} c_{1 j}(x)-d_{1}(t) \\
\frac{\partial}{\partial t_{2}}(p \tilde{h})-\sum_{j=1}^{2} \frac{\partial p}{\partial x_{j}} c_{2 j}(t)-d_{2}(t) \\
-p(x)
\end{array}\right)
$$

where

$$
\begin{aligned}
c_{i j}(t) & =\int_{Y}\left(\delta_{i j}+\frac{\partial \pi^{j}}{\partial y_{i}}\right) \frac{h}{2} d y & i, j=1,2 \\
d_{i}(t) & =\int_{Y}\left(\frac{\partial \pi^{3}}{\partial y_{i}} \frac{h}{2}+\mu \frac{s_{i}}{h}\right) d y & i=1,2 .
\end{aligned}
$$

3.2.2. Convergence of the stresses on the lower boundary. The lower boundary $\Gamma_{-}$has been supposed to be a flat surface, so it is no longer necessary to straighten the domain. It is sufficient to consider $\Gamma_{-}$as the lower boundary of the fixed subdomain $\Omega_{\min }$ of $\Omega^{\epsilon}$. We will only give the convergence results, the proofs of which are left to the reader.

TheOrem 3.5. For $\alpha=1$, the stress vector $V_{-}^{\epsilon}$ converges in $\left(H^{\frac{1}{2} 00}\left(\Gamma_{-}\right)\right)^{\prime 3}$ to $V_{-}^{\lambda}$, defined by

$$
V_{-}^{\lambda}=\left(\begin{array}{c}
\sum_{j=1}^{2} c_{1 j}^{\lambda} \frac{\partial p^{\lambda}}{\partial x_{j}}+d_{1}^{\lambda}(x) \\
\sum_{j=1}^{2} c_{2 j}^{\lambda} \frac{\partial p^{\lambda}}{\partial x_{j}}+d_{2}^{\lambda}(x) \\
\lambda^{2} p^{\lambda}
\end{array}\right)
$$

with

$$
c_{i j}^{\lambda}=\left(\frac{\partial \widetilde{w}_{i}^{j \lambda}}{\partial x_{3}}\right)_{x_{3}=0}, \quad d_{i}^{\lambda}=\left(\frac{\partial \widetilde{w}_{1}^{3 \lambda}}{\partial x_{3}}\right)_{x_{3}=0} \quad i, j=1,2,
$$

where $w^{j \lambda}(j=1,2,3)$ are the solutions to local problems (3.2) and (3.3).

For $\alpha>1, V_{-}^{\epsilon}$ converges in $\left(H^{\frac{1}{2}} 00\left(\Gamma_{-}\right)\right)^{\prime} 3$ to $V_{-}$, defined by

$$
V_{-}=\left(\begin{array}{c}
\sum_{j=1}^{2} c_{1 j}(x) \frac{\partial p}{\partial x_{j}}+d_{1}(x) \\
\sum_{j=1}^{2} c_{2 j}(x) \frac{\partial p}{\partial x_{j}} c_{2 j}(x)+d_{2}(x) \\
p(x)
\end{array}\right)
$$


with

$$
c_{i j}(x)=\int_{Y}\left(\delta_{i j}+\frac{\partial \pi^{j}}{\partial y_{i}}\right) \frac{h}{2 \mu} d y \quad i, j=1,2, \quad d_{i}=\int_{Y}\left(\frac{\partial \pi^{3}}{\partial y_{i}} \frac{h}{2 \mu}+\mu \frac{s_{i}}{h}\right) d y \quad i=1,2
$$

where $\pi^{k}(k=1,2,3)$ are the solutions to local problems defined in (3.5) and (3.6).

4. Proofs of the theorems in Section 3.1. In this section, we give the proofs of Theorem 3.1. Also, we will need some results for pressure and velocity. In the first paragraph, we give the estimates of the velocity and pressure in the domain $\Omega$; they are valid with the hypothesis $\eta \ll \epsilon$. The proofs of the main theorem are in Section 4.2 for the case of $\alpha=1$ and in Section 4.3 for the case of $\alpha>1$.

4.1. Asymptotic behaviour of the velocity and pressure for $\alpha \geq 1$. First, we give the estimates of the velocity in the rescaled domain $\Omega^{\epsilon}$. On the contrary, the pressure estimates are obtained in the fixed domain $\Omega$ and will be deduced in $\Omega^{\epsilon}$.

For any domain $D \subset \mathbb{R}^{3}$ we introduce the Hilbert space

$$
V_{x_{3}}(D)=\left\{v \in L^{2}(D), \frac{\partial v}{\partial x_{3}} \in L^{2}(D)\right\}
$$

equipped with the norm

$$
\|v\|_{V_{x_{3}}(D)}^{2}=\|v\|_{L^{2}(D)}^{2}+\left\|\frac{\partial v}{\partial x_{3}}\right\|_{L^{2}(D)}^{2} .
$$

Lemma 4.1. The following estimates are satisfied in $\Omega^{\epsilon}$ ( $K$ denotes any constant which does not rely on $\eta$ and $\epsilon$ ):

$$
\left\|u_{i}^{\epsilon}\right\|_{V_{x_{3}}\left(\Omega^{\epsilon}\right)} \leq K, \quad\left\|\eta \frac{\partial u_{i}^{\epsilon}}{\partial x_{j}}\right\|_{L^{2}\left(\Omega^{\epsilon}\right)^{3}} \leq K, \quad i=1,2,3, \quad j=1,2 .
$$

Proof. We take $u^{\epsilon}-G^{\epsilon}$ as a test function in (2.7). Since $\operatorname{div}_{\eta} G^{\epsilon}=0$,

$$
\int_{\Omega^{\epsilon}}\left(\nabla_{\eta} u^{\epsilon}\right)^{2} d x d x_{3}-\int_{\Omega^{\epsilon}} \nabla_{\eta} u^{\epsilon} \nabla_{\eta} G^{\epsilon} d x d x_{3}=0 .
$$

Using the Cauchy-Schwartz inequality, we obtain

$$
\left\|\nabla_{\eta} u^{\epsilon}\right\|_{L^{2}\left(\Omega^{\epsilon}\right)^{9}} \leq\left\|\nabla_{\eta} G^{\epsilon}\right\|_{L^{2}\left(\Omega^{\epsilon}\right)^{9}}=\left\|\nabla_{\eta} G^{\epsilon}\right\|_{L^{2}\left(\Omega_{m i n}\right)^{9}},
$$

so

$$
\left\|\nabla_{\eta} u^{\epsilon}\right\|_{L^{2}\left(\Omega^{\epsilon}\right)^{6}} \leq \frac{K}{\eta}
$$

This implies

$$
\left\|\eta \frac{\partial u^{\epsilon}}{\partial x_{i}}\right\|_{L^{2}\left(\Omega^{\epsilon}\right)^{3}} \leq K, i=1,2 \text { and }\left\|\frac{\partial u^{\epsilon}}{\partial x_{3}}\right\|_{L^{2}\left(\Omega^{\epsilon}\right)^{3}} \leq K .
$$

Using the Friedrich-Poincaré inequality in the $x_{3}$ direction for a function in $H^{1}\left(\Omega^{\epsilon}\right)$ equal to zero on $\Gamma_{+}^{\epsilon}$, we obtain (with $b$ defined in (2.1))

$$
\left\|u^{\epsilon}\right\|_{L^{2}\left(\Omega^{\epsilon}\right)^{3}} \leq b\left\|\frac{\partial u^{\epsilon}}{\partial x_{3}}\right\|_{L^{2}\left(\Omega^{\epsilon}\right)^{3}} \leq K .
$$

Now, we can deduce the estimates of velocity and pressure in the domain $\Omega$. 
Lemma 4.2. We have the following estimates for velocity and pressure:

$$
\begin{aligned}
\left\|u_{i}^{\epsilon}\right\|_{V_{t_{3}}(\Omega)} \leq K \quad \eta\left\|\frac{\partial u_{i}^{\epsilon}}{\partial t_{j}}\right\|_{L^{2}(\Omega)^{3}} & \leq K \quad i=1,2,3, \quad j=1,2 \\
\left\|\eta^{2} p^{\epsilon}\right\|_{L^{2}(\Omega)} & \leq K .
\end{aligned}
$$

Proof. Using (2.4) for the third variable,

$$
\int_{\Omega}\left(\frac{\partial u^{\epsilon}}{\partial t_{3}}\right)^{2} d t d t_{3}=\int_{\Omega^{\epsilon}}\left(\frac{\partial u^{\epsilon}}{\partial x_{3}}\right)^{2} h^{\epsilon} d x d x_{3} \leq K .
$$

Using the Poincare inequality in the $t_{3}$-direction in $\Omega$, as $u^{\epsilon}$ equals zero on the boundary $t_{3}=1$,

$$
\int_{\Omega}\left(u^{\epsilon}\right)^{2} d t d t_{3} \leq K \int_{\Omega}\left(\frac{\partial u^{\epsilon}}{\partial t_{3}}\right)^{2} d t d t_{3} \leq K
$$

Using (2.4) in the domain $\Omega$, we obtain

so

$$
\frac{\partial u^{\epsilon}}{\partial t_{i}}=\frac{\partial u^{\epsilon}}{\partial x_{i}}+\frac{\partial h^{\epsilon}}{\partial x_{i}} \frac{x_{3}}{h^{\epsilon}} \frac{\partial u^{\epsilon}}{\partial x_{3}}, \quad i=1,2,
$$

$$
\int_{\Omega}\left(\frac{\partial u^{\epsilon}}{\partial t_{i}}\right)^{2} d t d t_{3}=\int_{\Omega^{\epsilon}}\left(\frac{\partial u^{\epsilon}}{\partial x_{i}}+\frac{\partial h^{\epsilon}}{\partial x_{i}} \frac{x_{3}}{h^{\epsilon}} \frac{\partial u^{\epsilon}}{\partial x_{3}}\right)^{2} \frac{1}{h^{\epsilon}} d x d x_{3} \quad i=1,2 .
$$

Due to the oscillations of the function $h^{\epsilon}$, we obtain (formula (2.2))

$$
\left\|\frac{\partial h^{\epsilon}}{\partial x_{i}}\right\|_{L^{\infty}(w)} \leq \frac{K}{\epsilon}
$$

so that estimates from Lemma 4.1 imply

$$
\left\|\frac{\partial u^{\epsilon}}{\partial t_{i}}\right\|_{L^{2}(\Omega)} \leq \frac{K}{\eta} \quad i=1,2 .
$$

We now prove the estimates for the pressure. Taking $(0,0, \varphi)$ with $\varphi$ in $H_{0}^{1}(\Omega)$ in (2.10), we obtain

$$
\begin{array}{r}
\mu \int_{\Omega}\left[\sum_{i=1}^{2}\left(\frac{\partial}{\partial t_{i}}\left(\eta h^{\epsilon} u_{3}^{\epsilon}\right)+\frac{\partial}{\partial t_{3}}\left(-\eta t_{3} \frac{\partial h^{\epsilon}}{\partial t_{i}} u_{3}^{\epsilon}\right)\right)\left(\frac{\partial}{\partial t_{i}}\left(\eta h^{\epsilon} \varphi\right)+\frac{\partial}{\partial t_{3}}\left(-\eta t_{3} \frac{\partial h^{\epsilon}}{\partial t_{i}} \varphi\right)\right)\right. \\
\left.+\frac{\partial u_{3}^{\epsilon}}{\partial t_{3}} \frac{\partial \varphi}{\partial t_{3}}\right] \frac{d t d t_{3}}{\eta h^{\epsilon}}=\int_{\Omega} p^{\epsilon} \frac{\partial \varphi}{\partial t_{3}} d t d t_{3}
\end{array}
$$

The estimates of the velocity induce

$$
\left|\int_{\Omega} p^{\epsilon} \frac{\partial \varphi}{\partial t_{3}} d t d t_{3}\right| \leq \frac{K}{\eta}\|\varphi\|_{H_{0}^{1}(\Omega)} .
$$

On the other hand, taking $\left(\frac{\varphi}{h^{\epsilon}}, 0,0\right)$ in (2.10), we prove that

$$
\begin{array}{r}
I_{1}^{\epsilon}=\mu \int_{\Omega}\left[\sum_{i=1}^{2}\left(\frac{\partial}{\partial t_{i}}\left(\eta h^{\epsilon} u_{1}^{\epsilon}\right)+\frac{\partial}{\partial t_{3}}\left(-\eta t_{3} \frac{\partial h^{\epsilon}}{\partial t_{i}} u_{1}^{\epsilon}\right)\right) \cdot\left(\frac{\partial}{\partial t_{i}}(\eta \varphi)+\frac{\partial}{\partial t_{3}}\left(-\eta t_{3} \frac{1}{h^{\epsilon}} \frac{\partial h^{\epsilon}}{\partial t_{i}} \varphi\right)\right)\right. \\
\left.+\frac{1}{h^{\epsilon}} \frac{\partial u_{1}^{\epsilon}}{\partial t_{3}} \frac{\partial \varphi}{\partial t_{3}}\right] \frac{d t d t_{3}}{\eta h^{\epsilon}}=\int_{\Omega} p^{\epsilon}\left(\frac{\partial}{\partial t_{1}}(\eta \varphi)+\frac{\partial}{\partial t_{3}}\left(-\eta t_{3} \frac{\partial h^{\epsilon}}{\partial t_{1}} \frac{\varphi}{h^{\epsilon}}\right)\right) d t d t_{3}
\end{array}
$$


So we have

$$
\int_{\Omega} p^{\epsilon} \frac{\partial \varphi}{\partial t_{1}} d t d t_{3}=\frac{I_{1}^{\epsilon}}{\eta}+\int_{\Omega} p^{\epsilon} \frac{\partial}{\partial t_{3}}\left(t_{3} \frac{\partial h^{\epsilon}}{\partial t_{1}} \frac{\varphi}{h^{\epsilon}}\right) d t d t_{3} .
$$

Using the estimates of the velocity, we obtain

$$
\left|I_{1}^{\epsilon}\right| \leq \frac{K}{\eta}\|\varphi\|_{H_{0}^{1}(\Omega)}
$$

Taking now $\left(0,0, t_{3} \frac{\partial h^{\epsilon}}{\partial t_{1}} \frac{\varphi}{h^{\epsilon}}\right)$ in (2.10), we obtain

$$
\begin{gathered}
\mu \sum_{i=1}^{2} \int_{\Omega}\left(\frac{\partial}{\partial t_{i}}\left(\eta h^{\epsilon} u_{3}^{\epsilon}\right)+\frac{\partial}{\partial t_{3}}\left(-\eta t_{3} \frac{\partial h^{\epsilon}}{\partial t_{i}} u_{3}^{\epsilon}\right)\right) \\
\cdot\left(\frac{\partial}{\partial t_{i}}\left(\eta t_{3} \frac{\partial h^{\epsilon}}{\partial t_{1}} \varphi\right)+\frac{\partial}{\partial t_{3}}\left(-\eta \frac{t_{3}^{2}}{h^{\epsilon}} \frac{\partial h^{\epsilon}}{\partial t_{i}} \frac{\partial h^{\epsilon}}{\partial t_{1}} \varphi\right)\right) \frac{d t d t_{3}}{\eta h^{\epsilon}} \\
+\mu \int_{\Omega} \frac{1}{\eta h^{\epsilon}} \frac{\partial u_{3}^{\epsilon}}{\partial t_{3}} \frac{\partial}{\partial t_{3}}\left(t_{3} \frac{\partial h^{\epsilon}}{\partial t_{1}} \frac{\varphi}{h^{\epsilon}}\right) d t d t_{3} \\
=\int_{\Omega} p^{\epsilon} \frac{\partial}{\partial t_{3}}\left(t_{3} \frac{\partial h^{\epsilon}}{\partial t_{1}} \frac{\varphi}{h^{\epsilon}}\right) d t d t_{3},
\end{gathered}
$$

which leads to

$$
\left|\int_{\Omega} p^{\epsilon} \frac{\partial}{\partial t_{3}}\left(t_{3} \frac{\partial h^{\epsilon}}{\partial t_{k}} \frac{\varphi}{h^{\epsilon}}\right)\right| \leq \frac{K}{\eta^{2}}\|\varphi\|_{H_{0}^{1}(\Omega)} \quad k=1,2 .
$$

So

$$
\left|\int_{\Omega} p^{\epsilon} \frac{\partial \varphi}{\partial t_{1}} d t d t_{3}\right| \leq \frac{K}{\eta^{2}}\|\varphi\|_{H_{0}^{1}(\Omega)} .
$$

In the same way, we prove that

$$
\left|\int_{\Omega} p^{\epsilon} \frac{\partial \varphi}{\partial t_{2}} d t d t_{3}\right| \leq \frac{K}{\eta^{2}}\|\varphi\|_{H_{0}^{1}(\Omega)} .
$$

Using (4.4) and (4.6), this induces

$$
\left\|\frac{\partial p^{\epsilon}}{\partial t_{i}}\right\|_{H^{-1}(\Omega)} \leq \frac{K}{\eta^{2}} \quad(i=1,2) \quad\left\|\frac{\partial p^{\epsilon}}{\partial t_{3}}\right\|_{H^{-1}(\Omega)} \leq \frac{K}{\eta} .
$$

$p^{\epsilon}$ belongs to $L_{0}^{2}\left(\Omega^{\epsilon}\right)$, so

$$
0=\int_{\Omega^{\epsilon}} p^{\epsilon} d x d x_{3}=\int_{\Omega} p^{\epsilon} h^{\epsilon} d t d t_{3} .
$$

From [22], $h^{\epsilon} p^{\epsilon}$ being in $L_{0}^{2}\left(\Omega^{\epsilon}\right), \phi^{\epsilon}$ in $H_{0}^{1}(\Omega)^{3}$ exists with $h^{\epsilon} p^{\epsilon}=\operatorname{div} \phi^{\epsilon}$ and

$$
\left\|\phi^{\epsilon}\right\|_{H_{0}^{1}(\Omega)^{3}} \leq K\left\|\operatorname{div} \phi^{\epsilon}\right\|_{L^{2}(\Omega)}=K\left\|h^{\epsilon} p^{\epsilon}\right\|_{L^{2}(\Omega)},
$$

where $K$ is a constant with respect to $\epsilon$. So we obtain

$$
\int_{\Omega} p^{\epsilon}\left(h^{\epsilon} p^{\epsilon}\right) d t d t_{3}=\int_{\Omega} p^{\epsilon}\left(\frac{\partial \phi_{1}^{\epsilon}}{\partial t_{1}}+\frac{\partial \phi_{2}^{\epsilon}}{\partial t_{2}}+\frac{\partial \phi_{3}^{\epsilon}}{\partial t_{3}}\right) d t d t_{3}=-\sum_{i=1}^{3}<\frac{\partial p^{\epsilon}}{\partial t_{i}}, \phi_{i}^{\epsilon}>_{H^{-1}(\Omega), H_{0}^{1}(\Omega)} .
$$

Using (2.1) and estimates (4.8),

$$
a\left\|p^{\epsilon}\right\|_{L^{2}(\Omega)}^{2} \leq \int_{\Omega} h^{\epsilon}\left(p^{\epsilon}\right)^{2} d t d t_{3} \leq \frac{K}{\eta^{2}}\left\|\phi^{\epsilon}\right\|_{H_{0}^{1}(\Omega)^{3}} \leq \frac{K}{\eta^{2}}\left\|h^{\epsilon} p^{\epsilon}\right\|_{L^{2}(\Omega)} \leq \frac{K b}{\eta^{2}}\left\|p^{\epsilon}\right\|_{L^{2}(\Omega)},
$$


so we obtain

$$
\left\|p^{\epsilon}\right\|_{L^{2}(\Omega)} \leq \frac{K}{\eta^{2}}
$$

which completes the proof.

We present a technical lemma in two-scale convergence that will be useful for comparing different limits in the different domains.

LEMMA 4.3. Let $f^{\epsilon}$ be in $L^{2}\left(\Omega^{\epsilon}\right)$ and $\bar{f}^{\epsilon}$ its extension by zero to $\Omega_{\max }$. Suppose that $f^{\epsilon}$ (respectively $\bar{f}^{\epsilon}$ ) two-scale converge to $f_{1}$ in $\Omega \times[0,1]^{3}$ (respectively to $f_{2}$ in $\Omega_{\max } \times[0,1]^{3}$ ). If $f_{1}$ and $f_{2}$ do not rely on $y_{3}$, then

$$
f_{2}\left(x, x_{3}, y\right)=\left\{\begin{array}{l}
f_{1}\left(x, \frac{x_{3}}{h(x, y)}, y\right) \text { for } x_{3}<h(x, y) \\
0 \quad \text { elsewhere }
\end{array}\right.
$$

Proof. We choose $\varphi$ in $\mathcal{D}\left(\Omega_{\max }, \mathcal{C}_{\#}^{\infty}\left([0,1]^{3}\right)\right)$ with $\varphi=0$ for $x_{3} \leq h(x, y)$, and we obtain

$$
\begin{gathered}
0=\int_{\Omega_{\max }} \bar{f}^{\epsilon}\left(x, x_{3}\right) \varphi\left(x, x_{3}, \frac{x}{\epsilon}\right) d x d x_{3} \rightarrow \int_{\Omega_{\max } \times[0,1]^{3}} f_{2}\left(x, x_{3}, y\right) \varphi\left(x, x_{3}, y\right) d x d x_{3} d y \\
=\int_{\omega} \int_{Y} \int_{h(x, y)}^{h_{\max }} f_{2} \varphi\left(x, x_{3}, y\right) d x d y d x_{3},
\end{gathered}
$$

which shows that $f_{2}=0$ for $x_{3}>h(x, y)$.

Now we show that $f_{2}\left(x, x_{3}, y\right)=f_{1}\left(x, \frac{x_{3}}{h}, y\right)$ for $x_{3} \leq h(x, y)$. Let $\psi$ be in $\mathcal{D}\left(\Omega, \mathcal{C}_{\#}^{\infty}(Y)\right)$. The change of variables (2.4) induces

$$
\int_{\Omega} f^{\epsilon} \psi^{\epsilon}\left(t, t_{3}\right) d t d t_{3}=\int_{\Omega^{\epsilon}} f^{\epsilon} \psi^{\epsilon}\left(x, \frac{x_{3}}{h^{\epsilon}}\right) \frac{1}{h^{\epsilon}} d x d x_{3} .
$$

In the second integral, we extended by zero to $\Omega_{\max }$ and after passing to the two-scale limits in all terms, we obtain

$$
\int_{\Omega} \int_{Y} f_{1}\left(t, t_{3}, y\right) \psi\left(t, t_{3}, y\right) d t d t_{3} d y=\int_{\Omega_{\max }} \int_{Y} f_{2}\left(x, x_{3}, y\right) \psi\left(x, \frac{x_{3}}{h}, y\right) \frac{1}{h} d x d x_{3} d y \text {. }
$$

As $f_{2}=0$ for $x_{3}>h(x, y)$, the term on the right-hand side in (4.9) becomes

$$
\int_{\omega} \int_{Y} \int_{0}^{h(x, y)} f_{2}\left(x, x_{3}, y\right) \psi\left(x, \frac{x_{3}}{h}, y\right) \frac{1}{h} d x_{3} d y d x .
$$

Coming back to variables $\left(x, x_{3}, y\right)$ in (4.9),

$$
\begin{aligned}
\int_{\Omega} \int_{Y} f_{1}\left(t, t_{3}, y\right) \psi\left(t, t_{3}, y\right) d t d t_{3} d y & =\int_{\omega} \int_{Y} \int_{0}^{h} f_{1}\left(x, \frac{x_{3}}{h}, y\right) \psi\left(x, \frac{x_{3}}{h}, y\right) \frac{1}{h} d x d y d x_{3} \\
& =\int_{\omega} \int_{Y} \int_{0}^{h} f_{2}\left(x, x_{3}, y\right) \psi\left(x, \frac{x_{3}}{h}, y\right) \frac{1}{h} d y d x_{3} d x
\end{aligned}
$$

This ends the proof.

LEMMA 4.4. $p^{\lambda}(t)$ in $L^{2}(\omega)$ exists such that in $\Omega, \eta^{2} p^{\epsilon}$ two-scale converges to $p^{\lambda}(t)$ with

$$
\int_{\omega} \tilde{h} p^{\lambda}(t) d t=0
$$


Proof. From the estimates of $p^{\epsilon}$ in Lemma 4.2, and the two-scale convergence results (see [3]), there is $p^{\lambda}\left(t, t_{3}, y, y_{3}\right)$ in $L^{2}(\Omega \times] 0,1\left[^{3}\right)$ and a subsequence of $\epsilon$ denoted also by $\epsilon$, such that

$$
\eta^{2} p^{\epsilon} \rightarrow p^{\lambda}\left(t, t_{3}, y, y_{3}\right) \quad \text { in } L^{2}(\Omega \times] 0,1\left[^{3}\right) .
$$

First, we will show that $p^{\lambda}$ does not rely on $y_{3}$. Let $\phi \in \mathcal{D}\left(\Omega, \mathcal{C}_{\#}^{\infty}\left([0,1]^{3}\right)\right)$. We set $\phi^{\epsilon}=\phi\left(t, t_{3}, \frac{t}{\epsilon}, \frac{t_{3}}{\epsilon}\right)$ and take $\left(0,0, \phi^{\epsilon}\right)$ as a test function in (2.10) such that

$$
\begin{array}{r}
J_{1}^{\epsilon}=\mu \int_{\Omega}\left[\sum_{i=1,2}\left(\frac{\partial}{\partial t_{i}}\left(\eta h^{\epsilon} u_{3}^{\epsilon}\right)+\frac{\partial}{\partial t_{3}}\left(-\eta t_{3} \frac{\partial h^{\epsilon}}{\partial t_{i}} u_{3}^{\epsilon}\right)\right) \cdot\left(\frac{\partial}{\partial t_{i}}\left(\eta h^{\epsilon} \phi^{\epsilon}\right)+\frac{\partial}{\partial t_{3}}\left(-\eta t_{3} \frac{\partial h^{\epsilon}}{\partial t_{i}} \phi^{\epsilon}\right)\right)\right. \\
\left.+\frac{\partial u_{3}^{\epsilon}}{\partial t_{3}} \frac{\partial \phi^{\epsilon}}{\partial t_{3}}\right] \frac{d t d t_{3}}{\eta h^{\epsilon}}=\int_{\Omega} p^{\epsilon}\left(\frac{\partial \phi}{\partial t_{3}}+\frac{1}{\epsilon} \frac{\partial \phi}{\partial y_{3}}\right) d t d t_{3} .
\end{array}
$$

Multiplying by $\eta^{2} \epsilon$ and using Lemma 4.2 we obtain

$$
\left|\int_{\Omega} \eta^{2} p^{\epsilon}\left(\epsilon \frac{\partial \phi}{\partial t_{3}}+\frac{\partial \phi}{\partial y_{3}}\right) d t d t_{3}\right|=\left|\eta^{2} \epsilon J_{1}^{\epsilon}\right| \leq K \eta
$$

Passing to the limit, we obtain

$$
\int_{\Omega} \int_{[0,1]^{3}} p^{\lambda} \frac{\partial \phi}{\partial y_{3}} d t d t_{3} d y d y_{3}=0
$$

which induces that $p^{\lambda}$ does not rely on $y_{3}$. Now we show that $p^{\lambda}$ does not rely on $t_{3}$. We choose $\phi$ in $\mathcal{D}\left(\Omega, \mathcal{C}_{\#}^{\infty}(Y)\right)$ and we take $\left(0,0, \phi^{\epsilon}\right)$ as a test function in (2.10) with $\phi^{\epsilon}=\phi\left(t, t_{3}, \frac{t}{\epsilon}\right)$ :

$$
\begin{aligned}
& \mu \int_{\Omega}\left[\sum_{i=1,2}\left(\frac{\partial}{\partial t_{i}}\left(\eta h^{\epsilon} u_{3}^{\eta \epsilon}\right)+\frac{\partial}{\partial t_{3}}\left(-\eta t_{3} \frac{\partial h^{\epsilon}}{\partial t_{i}} u_{3}^{\epsilon}\right)\right) \cdot\left(\frac{\partial}{\partial t_{i}}\left(\eta h^{\epsilon} \phi^{\epsilon}\right)+\frac{\partial}{\partial t_{3}}\left(-\eta t_{3} \frac{\partial h^{\epsilon}}{\partial t_{i}} \phi^{\epsilon}\right)\right)\right. \\
& \left.+\frac{\partial u_{3}^{\eta \epsilon}}{\partial t_{3}} \frac{\partial \phi^{\epsilon}}{h^{\epsilon} \partial t_{3}}\right] \frac{d t d t_{3}}{\eta h^{\epsilon}}=\int_{\Omega} p^{\epsilon} \frac{\partial \phi^{\epsilon}}{\partial t_{3}} d t d t_{3} .
\end{aligned}
$$

It is sufficient to multiply by $\eta^{2}$ to obtain

$$
\left|\int_{\Omega} \eta^{2} p^{\epsilon} \frac{\partial \phi^{\epsilon}}{\partial t_{3}} d t d t_{3}\right| \leq K \eta
$$

Passing to the limit, we obtain

$$
\int_{\Omega} \int_{Y} p^{\lambda} \frac{\partial \phi}{\partial t_{3}} d t d t_{3} d y=0 \quad \forall \phi \in \mathcal{D}\left(\Omega, \mathcal{C}_{\#}^{\infty}(Y)\right),
$$

which proves that $p^{\lambda}$ does not rely on $t_{3}$.

To show that $p^{\lambda}$ is independent of $y$, we take $\phi^{\epsilon}$ as above, and $\left(\frac{\phi^{\epsilon}}{h^{\epsilon}}, 0,0\right)$ as a test function in (2.10):

$$
\begin{array}{r}
\mu \int_{\Omega}\left[\sum_{i=1}^{2}\left(\frac{\partial}{\partial t_{i}}\left(\eta h^{\epsilon} u_{1}^{\epsilon}\right)+\frac{\partial}{\partial t_{3}}\left(-\eta t_{3} \frac{\partial h^{\epsilon}}{\partial t_{i}} u_{1}^{\epsilon}\right)\right) \cdot\left(\frac{\partial}{\partial t_{i}}\left(\eta \phi^{\epsilon}\right)+\frac{\partial}{\partial t_{3}}\left(-\eta \frac{t_{3}}{h^{\epsilon}} \frac{\partial h^{\epsilon}}{\partial t_{i}} \phi^{\epsilon}\right)\right)\right. \\
\left.+\frac{\partial u_{1}^{\epsilon}}{\partial t_{3}} \frac{\partial \phi^{\epsilon}}{\partial t_{3}} \frac{1}{h^{\epsilon}}\right] \frac{d t d t_{3}}{\eta h^{\epsilon}}=\int_{\Omega} p^{\epsilon}\left(\frac{\partial}{\partial t_{1}}(\eta \phi)+\frac{1}{\epsilon} \frac{\partial}{\partial y_{1}}(\eta \phi)+\frac{\partial}{\partial t_{3}}\left(-\eta \frac{t_{3}}{h^{\epsilon}} \frac{\partial h^{\epsilon}}{\partial t_{1}} \phi\right)\right) d t d t_{3} .
\end{array}
$$


Multipying by $\eta \epsilon$ and passing to the limit, we obtain

$$
\int_{\Omega} \int_{Y} p^{\lambda} \frac{\partial \phi}{\partial y_{1}} d t d t_{3} d y-\int_{\Omega} \int_{Y} p^{\lambda} \frac{\partial}{\partial t_{3}}\left(\frac{t_{3}}{h} \frac{\partial h}{\partial y_{1}} \phi\right) d t d t_{3} d y=0 .
$$

By density we can use (4.11) to obtain

$$
\int_{\Omega} \int_{Y} p^{\lambda} \frac{\partial}{\partial t_{3}}\left(\frac{t_{3}}{h} \frac{\partial h}{\partial y} \phi\right) d t d t_{3} d y=0
$$

so that

$$
\int_{\Omega} \int_{Y} p^{\lambda} \frac{\partial \phi}{\partial y_{1}} d t d t_{3} d y=0
$$

Proceeding in the same way for $y_{2}$, we deduce that $p^{\lambda}$ does not rely on $y$.

Proposition 4.5. There is $\xi$ in $L^{2}\left(\Omega_{\max } \times Y\right)$ such that $\eta^{2} \bar{p}^{\epsilon}$ two-scale converges to $\xi\left(x, x_{3}, y\right)$ with

$$
\xi\left(x, x_{3}, y\right)= \begin{cases}p^{\lambda}(x) & \forall x_{3}<h(x, y) \\ 0 & \forall x_{3} \geq h(x, y) .\end{cases}
$$

Proof. Using the estimate given in Lemma 4.2, we obtain

$$
\eta^{2}\left\|\bar{p}^{\epsilon}\right\|_{L^{2}\left(\Omega_{\max }\right)}=\eta^{2}\left\|p^{\epsilon}\right\|_{L^{2}\left(\Omega^{\epsilon}\right)}=\eta^{2}\left(\int_{\Omega}\left(p^{\epsilon}\right)^{2} h^{\epsilon} d t d t_{3}\right)^{\frac{1}{2}} \leq K .
$$

So we can extract a subsequence such that $\eta^{2} \bar{p}^{\epsilon}$ converges weakly to $\xi\left(t, t_{3}, y, y_{3}\right)$ in $L^{2}\left(\Omega_{\max } \times[0,1]^{3}\right)$.

Now we show that $\xi$ does not rely on $y_{3}$. For any $\varphi$ in $\mathcal{D}\left(\Omega_{\max }, \mathcal{C}_{\#}^{\infty}\left([0,1]^{3}\right)\right)$ with $\varphi=0$ for $x_{3} \geq h(x, y)$, taking $\varphi^{\epsilon}\left(x, x_{3}\right)=\varphi\left(x, x_{3}, \frac{x}{\epsilon}\right),(0,0, \varphi)$ is a test function in (2.7).

$$
\begin{aligned}
I_{1}^{\epsilon}=\mu \int_{\Omega^{\epsilon}}\left[\sum_{i=1}^{2} \frac{\partial u_{3}^{\epsilon}}{\partial x_{i}}\left(\frac{\partial \varphi}{\partial x_{i}}+\frac{1}{\epsilon} \frac{\partial \varphi}{\partial y_{i}}\right)\right. & \left.+\frac{1}{\eta^{2}} \frac{\partial u_{3}^{\epsilon}}{\partial x_{3}}\left(\frac{\partial \varphi}{\partial x_{3}}+\frac{1}{\epsilon} \frac{\partial \varphi}{\partial y_{3}}\right)\right] d x d x_{3} \\
& =\frac{1}{\eta} \int_{\Omega^{\epsilon}} p^{\epsilon}\left(\frac{\partial \varphi}{\partial x_{3}}+\frac{1}{\epsilon} \frac{\partial \varphi}{\partial y_{3}}\right) d x d x_{3} .
\end{aligned}
$$

Extending $u^{\epsilon}$ and $p^{\epsilon}$ by zero to $\Omega_{\max }$ and passing to the limit after multiplication by $\eta^{3} \epsilon$, we obtain

$$
\int_{\Omega_{\max }} \int_{[0,1]^{3}} \xi \frac{\partial \varphi}{\partial y_{3}} d x d x_{3} d y d y_{3}=0
$$

To show that $\xi=0$ for $x_{3} \geq h(x, y)$, we choose $\varphi$ in $\mathcal{D}\left(\Omega_{\max }, \mathcal{C}_{\#}^{\infty}(Y)\right)$ with $\varphi=0$ for $x_{3} \leq h(x, y)$, we obtain

$$
\int_{\Omega_{\max }} \eta^{2} \bar{p}^{\epsilon} \varphi\left(x, x_{3}, \frac{x}{\epsilon}\right) d x d x_{3}=0 .
$$

Passing to the two-scale limit, we obtain the result. Lemma 4.3 completes the proof. 
4.2. Proof of Theorem 3.1. In this section we prove Theorem 3.1 which specifies the limit pressure in the case $\alpha=1$, which corresponds to

$$
\eta=\lambda \epsilon
$$

To this topic, we first give the two-scale convergence result for the velocity, and then the relation between limit pressure and velocity involving the local problems.

Proposition 4.6. There is $u^{\lambda}$ in $\left(L^{2}\left(\Omega_{\text {max }} ; H_{p e r}^{1}(Y)\right)\right)^{3}$ with $\frac{\partial u^{\lambda}}{\partial x_{3}}$ in $\left(L^{2}\left(\Omega_{\max } \times Y\right)\right)^{3}$ and there is $v^{\lambda}$ in $\left(L^{2}\left(\Omega_{\max } \times Y ; H_{p e r}^{1}([0,1])\right)\right)^{3}$ such that

$$
\begin{gathered}
\bar{u}^{\epsilon} \rightarrow u^{\lambda}, \\
\frac{\partial \bar{u}^{\epsilon}}{\partial x_{3}} \rightarrow \frac{\partial u^{\lambda}}{\partial x_{3}}+\frac{\partial v^{\lambda}}{\partial y_{3}}, \\
\epsilon \frac{\partial \bar{u}^{\epsilon}}{\partial x_{i}} \rightarrow \frac{\partial u^{\lambda}}{\partial y_{i}} \quad i=1,2 .
\end{gathered}
$$

Moreover,

$$
\begin{gathered}
\operatorname{div}_{\lambda} u^{\lambda}=\frac{\partial u_{1}^{\lambda}}{\partial y_{1}}+\frac{\partial u_{2}^{\lambda}}{\partial y_{2}}+\frac{1}{\lambda} \frac{\partial u_{3}^{\lambda}}{\partial x_{3}}=0 \text { in } B_{x} \text { a.e. } x \in \omega, \\
u^{\lambda}=0 \text { for } x_{3} \geq h(x, y) \text { and } u^{\lambda}=\left(s_{1}, s_{2}, 0\right) \text { for } x_{3}=0 .
\end{gathered}
$$

Proof. From estimates (4.2), we obtain

$$
\begin{gathered}
\left\|\bar{u}^{\epsilon}\right\|_{L^{2}\left(\Omega_{\max }\right)^{3}}=\left\|u^{\epsilon}\right\|_{L^{2}\left(\Omega^{\epsilon}\right)^{3}} \leq K, \\
\epsilon\left\|\frac{\partial \bar{u}^{\epsilon}}{\partial x_{i}}\right\|_{L^{2}\left(\Omega_{\max }\right)^{3}} \leq K, \quad i=1,2, \\
\left\|\frac{\partial \bar{u}^{\epsilon}}{\partial x_{3}}\right\|_{L^{2}\left(\Omega_{\max }\right)^{3}} \leq K .
\end{gathered}
$$

So applying the two-scale convergence results of Proposition 1.14 in [3], we deduce the existence of $u^{\lambda}$ in $L^{2}\left(\Omega_{\max } ; H_{p e r}^{1}[0,1]^{3}\right)^{3}$ and $v^{\lambda}$ in $\left(L^{2}\left(\Omega_{\max } \times Y ; H_{p e r}^{1}[0,1]\right)\right)^{3}$ such that

$$
\begin{gathered}
\bar{u}^{\epsilon} \rightarrow u^{\lambda}, \\
\frac{\partial \bar{u}^{\epsilon}}{\partial x_{3}} \rightarrow \frac{\partial u^{\lambda}}{\partial x_{3}}+\frac{\partial v^{\lambda}}{\partial y_{3}}, \\
\epsilon \frac{\partial \bar{u}^{\epsilon}}{\partial x_{i}} \rightarrow \frac{\partial u^{\lambda}}{\partial y_{i}} i=1,2 .
\end{gathered}
$$

Let us prove that $u^{\lambda}$ does not rely on $y_{3}$. Exactly as for the pressure, we obtain $u^{\lambda}=0$ for $x_{3} \geq h(x, y)$. Then, setting $\varphi^{\epsilon}=\varphi\left(x, x_{3}, \frac{x}{\epsilon}, \frac{x_{3}}{\epsilon}\right)$ with $\varphi$ in $\mathcal{D}\left(\Omega_{\max } \times[0,1]^{3}\right)^{3}$ and $\varphi=0$ for $x_{3} \geq h(x, y)$, we obtain

$$
\begin{aligned}
& \int_{\Omega^{\epsilon}} \frac{\partial u^{\epsilon}}{\partial x_{3}} \cdot \varphi^{\epsilon} d x d x_{3}=-\int_{\Omega^{\epsilon}} u^{\epsilon} \cdot\left(\frac{\partial \varphi}{\partial x_{3}}+\frac{1}{\epsilon} \frac{\partial \varphi}{\partial y_{3}}\right) d x d x_{3} \\
& =-\int_{\Omega_{\max }} \bar{u}^{\epsilon} \cdot\left(\frac{\partial \varphi}{\partial x_{3}}+\frac{1}{\epsilon} \frac{\partial \varphi}{\partial y_{3}}\right) d x d x_{3} .
\end{aligned}
$$


Passing to the limit in $\Omega_{\max }$ after multiplication by $\epsilon$, we obtain

$$
0=\int_{\Omega_{\max }} \int_{Y} u^{\lambda} \cdot \frac{\partial \varphi}{\partial y_{3}} d x d x_{3} d y
$$

which induces $\frac{\partial u^{\lambda}}{\partial y_{3}}=0$ for $x_{3}<h(x, y)$, so $u^{\lambda}$ does not rely on $y_{3}$.

Let $\varphi^{\epsilon}=\varphi\left(x, x_{3}, \frac{x}{\epsilon}\right)$ with $\varphi$ arbitrary in $\mathcal{D}\left(\Omega_{\text {max }} ; \mathcal{C}_{\#}^{\infty}(Y)\right)$. From (2.8), we obtain

$$
\int_{\Omega^{\epsilon}}\left(\frac{\partial u_{1}^{\epsilon}}{\partial x_{1}}+\frac{\partial u_{2}^{\epsilon}}{\partial x_{2}}+\frac{1}{\eta} \frac{\partial u_{3}^{\epsilon}}{\partial x_{3}}\right) \varphi^{\epsilon} d x d x_{3}=0
$$

Using the Green formula on each term, we obtain

$$
\int_{\Omega^{\epsilon}} u_{1}^{\epsilon}\left(\frac{\partial \varphi}{\partial x_{1}}+\frac{1}{\epsilon} \frac{\partial \varphi}{\partial y_{1}}\right) d x d x_{3}+\int_{\Omega^{\epsilon}} u_{2}^{\epsilon}\left(\frac{\partial \varphi}{\partial x_{2}}+\frac{1}{\epsilon} \frac{\partial \varphi}{\partial y_{2}}\right) d x d x_{3}+\frac{1}{\eta} \int_{\Omega^{\epsilon}} u_{3}^{\epsilon} \frac{\partial \varphi}{\partial x_{3}} d x d x_{3}=0 .
$$

Passing to the two-scale limit in $\Omega_{\max }$, after multiplication by $\epsilon$, we obtain

$$
\int_{Q}\left(u_{1}^{\lambda} \frac{\partial \varphi}{\partial y_{1}}+u_{2}^{\lambda} \frac{\partial \varphi}{\partial y_{2}}+\frac{1}{\lambda} u_{3}^{\lambda} \frac{\partial \varphi}{\partial x_{3}}\right) d x d y d x_{3}=\int_{Q} \operatorname{div}_{\lambda} u^{\lambda} \varphi d x d y d x_{3}=0
$$

with $Q$ defined in (3.1). Now taking $\varphi$ in the form

$$
\varphi\left(x, x_{3}, y\right)=\theta \psi_{x}\left(x_{3}, y\right)
$$

with $\theta$ and $\psi_{x}$ arbitrary in $\mathcal{D}(\omega)$ (respectively in $\mathcal{D}\left(B_{x}\right)$ ), we obtain the result. We now prove that the boundary conditions are still valid for the two-scale velocity limit. Passing to the limit in the Green formula

$$
\int_{\Omega_{\max }} \frac{\partial \bar{u}^{\epsilon}}{\partial x_{3}} \cdot \varphi\left(x, \frac{x}{\epsilon}, x_{3}\right) d x d x_{3}=-\int_{\Omega_{\max }} \bar{u}^{\epsilon} \cdot \frac{\partial \varphi}{\partial x_{3}} d x d x_{3}+\int_{\omega}\left(s_{1}, s_{2}, 0\right) \cdot \varphi\left(x, \frac{x}{\epsilon}, 0\right) d x
$$

for any $\varphi$ in $C^{\infty}\left(\Omega_{\max }, C_{\#}^{\infty}(Y)\right)$, we obtain

$$
\begin{array}{r}
\int_{\Omega_{\max }} \int_{Y} \frac{\partial \bar{u}^{\lambda}}{\partial x_{3}} \cdot \varphi\left(x, y, x_{3}\right) d x d y d x_{3} \\
=-\int_{\Omega_{\max }} \int_{Y} \bar{u}^{\lambda} \cdot \frac{\partial \varphi}{\partial x_{3}} d x d y d x_{3}+\int_{\omega} \int_{Y}\left(s_{1}, s_{2}, 0\right) \cdot \varphi(x, y, 0) d x d y .
\end{array}
$$

Applying again the Green formula, it is obvious that

$$
u^{\lambda}(x, y, 0)=\left(s_{1}, s_{2}, 0\right) \quad \text { a.e. }(x, y) \in \omega \times Y .
$$

Now, it is necessary to use the local problems (3.2), (3.3) in order to give a relation between the limit velocity and pressure.

Proposition 4.7. The limit pressure $p^{\lambda}$ belongs to $H^{1}(\omega)$ and the following relation is satisfied:

$$
u^{\lambda}=\sum_{j=1}^{2} \frac{\partial p^{\lambda}}{\partial x_{j}} w^{j \lambda}+w^{3 \lambda}
$$


Proof. The proof needs two steps.

Step 1: We will prove the relation

$\mu \int_{w} \int_{B_{x}}\left[\sum_{i=1}^{2} \frac{\partial u^{\lambda}}{\partial y_{i}} \cdot \frac{\partial \varphi}{\partial y_{i}}+\frac{1}{\lambda^{2}} \frac{\partial u^{\lambda}}{\partial x_{3}} \cdot \frac{\partial \varphi}{\partial x_{3}}\right] d x d x_{3} d y=\int_{w} \int_{B_{x}} p^{\lambda}(x)\left(\frac{\partial \varphi_{1}}{\partial x_{1}}+\frac{\partial \varphi_{2}}{\partial x_{2}}\right) d x d x_{3} d y$

for any $\varphi$ in $\left(\mathcal{D}\left(\Omega_{\text {max }} ; C_{\#}^{\infty}(Y)\right)^{3}\right.$ with $\varphi=0$ for $x_{3} \geq h(x, y)$ and $\operatorname{div}{ }_{\lambda} \varphi=0$. We set $\varphi^{\epsilon}\left(x, x_{3}\right)=\varphi\left(x, \frac{x}{\epsilon}, x_{3}\right)$ which can be chosen as a test function in (2.7):

$$
\begin{aligned}
& \mu \int_{\Omega_{\max }}\left[\sum_{i=1}^{2} \frac{\partial \bar{u}^{\epsilon}}{\partial x_{i}} \cdot\left(\frac{\partial \varphi}{\partial x_{i}}+\frac{1}{\epsilon} \frac{\partial \varphi}{\partial y_{i}}\right)+\frac{1}{\lambda^{2} \epsilon^{2}} \frac{\partial \bar{u}^{\epsilon}}{\partial x_{3}} \cdot \frac{\partial \varphi}{\partial x_{3}}\right] d x d x_{3} \\
& \quad=\int_{\Omega_{\max }} \bar{p}^{\epsilon}\left(\frac{\partial \varphi_{1}}{\partial x_{1}}+\frac{1}{\epsilon} \frac{\partial \varphi_{1}}{\partial y_{1}}+\frac{\partial \varphi_{2}}{\partial x_{2}}+\frac{1}{\epsilon} \frac{\partial \varphi_{2}}{\partial y_{2}}+\frac{1}{\lambda \epsilon} \frac{\partial \varphi_{3}}{\partial x_{3}}\right) d x d x_{3}
\end{aligned}
$$

As $\operatorname{div}_{\lambda} \varphi=0$, we obtain

$$
\begin{gathered}
\mu \int_{\Omega_{\max }}\left[\sum_{i=1}^{2} \frac{\partial \bar{u}^{\epsilon}}{\partial x_{i}} \cdot\left(\frac{\partial \varphi}{\partial x_{i}}+\frac{1}{\epsilon} \frac{\partial \varphi}{\partial y_{i}}\right)+\frac{1}{\lambda^{2} \epsilon^{2}} \frac{\partial \bar{u}^{\epsilon}}{\partial x_{3}} \cdot \frac{\partial \varphi}{\partial x_{3}}\right] d x d x_{3} \\
=\int_{\Omega_{\max }} \bar{p}^{\epsilon}\left(\frac{\partial \varphi_{1}}{\partial x_{1}}+\frac{\partial \varphi_{2}}{\partial x_{2}}\right) d x d x_{3} .
\end{gathered}
$$

Passing to the limit after multiplication by $\epsilon^{2}$, we obtain

$$
\begin{gathered}
\mu \int_{\Omega_{\max }} \int_{Y} \sum_{i=1}^{2} \frac{\partial u^{\lambda}}{\partial y_{i}} \cdot \frac{\partial \varphi}{\partial y_{i}} d x d x_{3} d y+\int_{\Omega_{\max }} \int_{Y} \frac{1}{\lambda^{2}}\left(\frac{\partial u^{\lambda}}{\partial x_{3}}+\int_{Y} \frac{\partial v^{\lambda}}{\partial y_{3}} d y_{3}\right) \cdot \frac{\partial \varphi}{\partial x_{3}} d x d x_{3} d y \\
=\int_{\Omega_{\max }} \int_{Y} p^{\lambda}(x)\left(\frac{\partial \varphi_{1}}{\partial x_{1}}+\frac{\partial \varphi_{2}}{\partial x_{2}}\right) d x d x_{3} d y
\end{gathered}
$$

Using the periodicity of $v^{\lambda}$ and the fact that $u^{\lambda}=0$ for $x_{3} \geq h(x, y)$, we obtain (4.13).

Step 2: We now prove that $p^{\lambda} \in H^{1}(\omega)$ and that $u^{\lambda}$ satisfies a Stokes equation in $B_{x}$, which will lead to 4.25).

Let $\psi=\psi\left(y_{2}, x_{3}\right)$ be a function that does not rely on $y_{1}$ in $C_{0 \#}^{\infty}(] 0,1[\times] 0, h_{\min }[)$. We denote by $\bar{\psi}$ its extension by zero in $B_{x}$. We suppose that $\tilde{\psi}=\int_{B_{x}} \bar{\psi} d x_{3} d y \neq 0$. Let $\phi=\left(\frac{\theta \bar{\psi}}{\tilde{\psi}}, 0,0\right)$ with $\theta$ in $\mathcal{D}(w)$. As $\operatorname{div}_{\lambda} \phi=0, \phi$ is a test function in (4.13), so

$$
\mu \int_{w} \int_{B_{x}} \nabla_{\lambda} u^{\lambda} \cdot \nabla_{\lambda} \phi d x d x_{3} d y=\int_{w} \int_{B_{x}} p^{\lambda}(x) \frac{\partial}{\partial x_{1}}\left(\theta \frac{\bar{\psi}}{\tilde{\psi}}\right) d x d x_{3} d y .
$$

As $\theta$ and $p^{\lambda}$ do not rely on variables $y, x_{3}$, we obtain

$$
\frac{\mu}{\tilde{\psi}} \int_{w} \theta(x)\left(\int_{B_{x}} \nabla_{\lambda} u_{1}^{\lambda} \nabla_{\lambda} \bar{\psi} d x_{3} d y\right) d x=\int_{w} p^{\lambda} \frac{\partial \theta}{\partial x_{1}}\left(\int_{B_{x}} \bar{\psi} \frac{1}{\tilde{\psi}} d x_{3} d y\right) d x=\int_{w} p^{\lambda} \frac{\partial \theta}{\partial x_{1}} d x .
$$

As $\nabla_{\lambda} u_{1}^{\lambda}$ belongs to $L^{2}(Q)^{3},\left(\int_{B_{x}} \nabla_{\lambda} u_{1}^{\lambda} \nabla_{\lambda} \bar{\psi} d x_{3} d y\right)$ lies in $L^{2}(w)$, which implies that $\frac{\partial p^{\lambda}}{\partial x_{1}}$ is in $L^{2}(w)$. 
In the same way, we prove that $\frac{\partial p^{\lambda}}{\partial x_{2}}$ is in $L^{2}(w)$, so that $p^{\lambda}$ belongs to $H^{1}(w)$. Now we choose in (4.13) $\psi=\theta(x) \cdot \varphi_{x}\left(y, x_{3}\right)$ with $\theta$ in $\mathcal{D}(w), \varphi_{x}$ in $\left(C_{0 \#}^{\infty}\left(B_{x}\right)\right)^{3}$, and $\operatorname{div}_{\lambda} \varphi=0$ :

$$
\begin{gathered}
\mu \int_{w} \theta\left(\int_{B_{x}} \nabla_{\lambda} u^{\lambda} \cdot \nabla_{\lambda} \varphi d x_{3} d y\right) d x=\int_{w} \int_{B_{x}} p^{\lambda}\left(\frac{\partial}{\partial x_{1}}(\theta \varphi)_{1}+\frac{\partial}{\partial x_{2}}(\theta \varphi)_{2}\right) d x d x_{3} d y \\
=-\int_{w}\left[\int_{B_{x}}\left(\frac{\partial p^{\lambda}}{\partial x_{1}} \varphi_{1}+\frac{\partial p^{\lambda}}{\partial x_{2}} \varphi_{2}\right) d x_{3} d y\right] \theta d x .
\end{gathered}
$$

This is true for all $\theta$ in $\mathcal{D}(w)$, and by the density argument and Proposition 4.6, we obtain the following Stokes problem:

$$
\begin{array}{r}
\mu \int_{B_{x}} \nabla_{\lambda} u^{\lambda} \cdot \nabla_{\lambda} \varphi d x_{3} d y=-\int_{B_{x}}\left(\varphi_{1} \frac{\partial p^{\lambda}}{\partial x_{1}}+\varphi_{2} \frac{\partial p^{\lambda}}{\partial x_{2}}\right) d x_{3} d y \text { a.e. } x \in w . \\
\forall \varphi \in\left\{\left(H_{0 \#}^{1}\left(B_{x}\right)\right)^{3}, \operatorname{div}_{\lambda} \varphi=0\right\}
\end{array}
$$

and

$$
\operatorname{div}_{\lambda} u^{\lambda}=0 \text { a.e. } x \in \omega .
$$

Since $p^{\lambda}$ depends only on $x$, we obtain by linearity and using the local problems (3.2), (3.3) the desired relation.

Proof of Theorem 3.1. Choosing in (2.8) $\varphi \in H^{1}(\omega)$ and using the Green formula in each term, we obtain

$$
-\int_{\Omega^{\epsilon}} u_{1}^{\epsilon} \frac{\partial \varphi}{\partial x_{1}} d x d x_{3}+\int_{\partial \Omega^{\epsilon}} u_{1}^{\epsilon} n_{1} \varphi d \sigma-\int_{\Omega^{\epsilon}} u_{2}^{\epsilon} \frac{\partial \varphi}{\partial x_{2}} d x d x_{3}+\int_{\partial \Omega^{\epsilon}} u_{2}^{\epsilon} n_{2} \varphi d \sigma=0 .
$$

Using the boundary conditions on the velocity $u^{\epsilon}$, we obtain

$$
\int_{\Omega^{\epsilon}} u_{1}^{\epsilon} \frac{\partial \varphi}{\partial x_{1}} d x d x_{3}+\int_{\Omega^{\epsilon}} u_{2}^{\epsilon} \frac{\partial \varphi}{\partial x_{2}} d x d x_{3}=\int_{\Gamma_{l}} g \cdot n \varphi d \sigma .
$$

Passing to the limit yields

$$
\int_{w} \int_{B_{x}}\left(u_{1}^{\lambda} \frac{\partial \varphi}{\partial x_{1}}+u_{2}^{\lambda} \frac{\partial \varphi}{\partial x_{2}}\right) d x d x_{3}=\int_{\partial w} f \cdot n \varphi d \sigma \quad \forall \varphi \in H^{1}(w)
$$

with

$$
f=\int_{0}^{h_{\min }} g d x_{3} .
$$

Now we will use the expression (4.12) of the velocity in (4.16) in order to identify the limit equation for $p^{\lambda}$ :

$$
\int_{w} \int_{B_{x}} \sum_{i=1}^{2}\left(\sum_{j=1}^{2} \frac{\partial p^{\lambda}}{\partial x_{j}} w_{i}^{j \lambda}+w_{i}^{3 \lambda}\right) \frac{\partial \varphi}{\partial x_{i}}=\int_{\partial w} f . n \varphi d \sigma \quad \forall \varphi \in H^{1}(w) .
$$

As $p^{\lambda}$ and $\varphi$ do not rely on variables $y$ and $x_{3}$, this equation gives (3.4).

It remains to prove the uniqueness of the solution of (3.4). For this it is sufficient to prove that the matrix $A^{\lambda}$ whose entries $a_{i j}^{\lambda}(x)$ are defined in Theorem 3.1 is symmetric and positive definite. 
Let us choose $w^{1 \lambda}\left(\right.$ resp. $\left.w^{2 \lambda}\right)$ as a test function in (3.2) with $k=2($ resp. $k=1)$ :

$$
\mu \int_{B_{x}} \nabla_{\lambda} w^{1 \lambda} \cdot \nabla_{\lambda} w^{2 \lambda} d y d x_{3}=-\int_{B_{x}} w_{1}^{2 \lambda}=a_{12}^{\lambda}=a_{21}^{\lambda},
$$

which proves the symmetry of $A^{\lambda}$. For arbitrary $\xi=\left(\xi_{1}, \xi_{2}\right)$ in $\mathbb{R}^{2}$, we choose $\xi_{i} \xi_{j} w^{j \lambda}$ as a test function in (3.2) with $k=i$ :

$$
\mu \xi_{i} \xi_{j} \int_{B_{x}} \nabla_{\lambda} w^{i \lambda} \cdot \nabla_{\lambda} w^{j \lambda} d x_{3} d y=-\int_{B_{x}} \xi_{i} \xi_{j} w_{i}^{j \lambda} d y d x_{3}=\xi_{i} \xi_{j} a_{i j}^{\lambda}
$$

By addition, this implies

$$
\sum_{i, j=1}^{2} \xi_{i} \xi_{j} a_{i j}^{\lambda}=\mu \int_{B_{x}}\left(\xi_{1} \nabla_{\lambda} w^{1 \lambda}+\xi_{2} \nabla_{\lambda} w^{2 \lambda}\right)^{2} d y d x_{3} \geq 0
$$

Suppose now that $\sum_{i, j=1}^{2} \xi_{i} \xi_{j} a_{i j}^{\lambda}=0$, that is

$$
\int_{B_{x}}\left(\nabla_{\lambda}\left(\xi_{1} w^{1 \lambda}+\xi_{2} w^{2 \lambda}\right)\right)^{2} d y d x_{3}=0
$$

By Poincaré's inequalities, this gives

$$
\xi_{1} w^{1 \lambda}+\xi_{2} w^{2 \lambda}=0
$$

From (3.2), taking successively $k=1$ and 2 , we obtain

$$
\int_{B_{x}}\left(\xi_{1} \varphi_{1}+\xi_{2} \varphi_{2}\right) d y d x_{3}=0
$$

for any $\varphi$ in $\left(H_{0 \#}^{1}\left(B_{x}\right)\right)^{3}$ with $\operatorname{div}_{\lambda}=0$. Now, taking $\varphi=\left(\varphi_{1}\left(y_{2}, x_{3}\right), 0,0\right)$ with $\varphi_{1} \in$ $H_{0 \#}^{1}(\omega \times] 0,1[)$ and $\int_{B_{x}} \varphi d y d x_{3} \neq 0$, we deduce $\xi_{1}=0$. In the same way, $\xi_{2}=0$, which ends the proof of Theorem 3.1

4.3. Proof of Theorem $3.2(\alpha>1)$. We recall that, in all these sections, the relation $\eta=\lambda \epsilon^{\alpha}$ with $\alpha>1$ is satisfied. The limit of the pressure and velocity will be denoted without the $\lambda$ superscript, since they will not depend on $\lambda$. Passing to the limit in the variational formulation in (2.7), only derivatives in $x_{3}$ will remain in the limit velocity term. Henceforth the limit $u$ and the test function are no longer in the same functional space and a density lemma is needed.

First, we introduce the following spaces:

$$
\begin{gathered}
V_{0}=\left\{\varphi=\left(\varphi_{1}, \varphi_{2}\right) \in\left(V_{x_{3}}\left(B_{x}\right)\right)^{2}, \varphi(x, 0)=\varphi(x, h)=0\right\}, \\
V_{00}=\left\{\varphi \in V_{0}, \int_{Y}\left(\int_{0}^{h} \varphi d x_{3}\right) \cdot \nabla_{Y} \psi d y=0 \quad \forall \psi \in H_{p e r}^{1}(Y)\right\}, \\
V=\left\{\left(\varphi_{1}, \varphi_{2}\right) \in\left(C_{0 \#}^{\infty}\left(B_{x}\right)\right)^{2} \quad \exists \varphi_{3} \in C_{0 \#}^{\infty}\left(B_{x}\right) \text { with } \frac{\partial \varphi_{1}}{\partial y_{1}}+\frac{\partial \varphi_{2}}{\partial y_{2}}+\frac{\partial \varphi_{3}}{\partial x_{3}}=0\right\} .
\end{gathered}
$$

It is easy to see that $V_{0}$ and $V_{00}$ are Hilbert spaces as they are closed subspaces of $\left(V_{x_{3}}\left(B_{x}\right)\right)^{2}$. We will need the following density lemma. 
LEMMA 4.8.

$$
\bar{V}=V_{00}
$$

where the adherence is in the sense of the norm of $\left(V_{x_{3}}\left(B_{x}\right)\right)^{2}$ (see (4.1)).

Proof. We first prove the inclusion $\bar{V} \subset V_{00}$. Let $\varphi$ be in $\bar{V}$. There is a sequence $\left(\varphi_{n}\right)=\left(\varphi_{1 n}, \varphi_{2 n}\right)$ in $V$ such that $\varphi_{n} \rightarrow \varphi$ in $\left(V_{x_{3}}\left(B_{x}\right)\right)^{2}$. So for any $n$ there exists $\varphi_{3 n}$ in $C_{0 \#}^{\infty}\left(B_{x}\right)$ such that for any $\psi \in H_{p e r}^{1}(Y)$, we obtain

$$
\int_{B_{x}}\left(\frac{\partial \varphi_{1 n}}{\partial y_{1}}+\frac{\partial \varphi_{2 n}}{\partial y_{2}}+\frac{\partial \varphi_{13}}{\partial x_{3}}\right) \psi d y d x_{3}=0
$$

Using the Green formula and the fact that $\psi$ is a function of $y$ only, we obtain

$$
\int_{Y}\left(\int_{0}^{h} \varphi_{n} d x_{3}\right) \nabla_{y} \psi d y=0
$$

which proves that $\varphi$ belongs to $V_{00}$ by passing to the limit in $n$.

To prove that $\bar{V}=V_{00}$, we will show, as in Theorem 1.6 page 18 of [22], that any continuous and linear form $L$ on $V_{00}$ vanishes on $\bar{V}$ is identically equal to zero. Let $L$ be a continuous form on $V_{00}$ that vanishes on $\bar{V}$. Applying the Riesz theorem, there is $l$ in $V_{00}$ such that

$$
L(v)=\sum_{i=1}^{2} \int_{B_{x}}\left(l_{i} v_{i}+\frac{\partial l_{i}}{\partial x_{3}} \frac{\partial v_{i}}{\partial x_{3}}\right) d y d x_{3} \quad \forall v \in V_{00} .
$$

So, we obtain

$$
\sum_{i=1}^{2} \int_{B_{x}}\left(l_{i} v_{i}+\frac{\partial l_{i}}{\partial x_{3}} \frac{\partial v_{i}}{\partial x_{3}}\right) d y d x_{3}=0 \quad \forall v \in V .
$$

The relation above is in fact true for any $\left(v_{1}, v_{2}, v_{3}\right)$ in $C_{0}^{\infty}\left(B_{x}\right)$ such that

$$
\frac{\partial v_{1}}{\partial y_{1}}+\frac{\partial v_{2}}{\partial y_{2}}+\frac{\partial v_{3}}{\partial x_{3}}=0
$$

Applying the De Rham theorem, we deduce by density the existence of $q$ in $L^{2}\left(B_{x}\right)$ such that

$$
\sum_{i=1}^{2} \int_{B_{x}}\left(l_{i} \phi_{i}+\frac{\partial l_{i}}{\partial x_{3}} \frac{\partial \phi_{i}}{\partial x_{3}}\right) d y d x_{3}=\int_{B_{x}} q \operatorname{div}_{B_{x}} \phi d y_{1} d y d x_{3} \quad \forall \phi \in\left(H_{0}^{1}\left(B_{x}\right)\right)^{3} .
$$

Choosing successively $\phi=(\varphi, 0,0), \phi=(0, \varphi, 0)$ and $\phi=(0,0, \varphi)$ with $\varphi$ in $H_{0}^{1}\left(B_{x}\right)$, we deduce from (4.19)

$$
l_{i}-\frac{\partial^{2} l_{i}}{\partial x_{3}^{2}}=-\frac{\partial q}{\partial y_{i}} \quad i=1,2 \quad \text { in } H^{-1}\left(B_{x}\right)
$$

and

$$
\int_{B_{x}} q \frac{\partial \psi}{\partial x_{3}} d y d x_{3}=0 \quad \forall \varphi \in H_{0}^{1}\left(B_{x}\right)
$$

which implies that $q$ does not rely on $x_{3}$. Let us now prove that $q$ lies in $H_{p e r}^{1}(Y)$. 
Set $\phi=\left(\frac{x_{3}}{h^{3}(x, y)}\left(x_{3}-h\right) \psi(y), 0,0\right)$ with $\psi \in H_{p e r}^{1}(Y)$ in (4.19):

$\int_{B_{x}} l_{1} \frac{x_{3}}{h^{3}}\left(x_{3}-h\right) \psi d y d x_{3}+\int_{B_{x}} \frac{\partial l_{1}}{\partial x_{3}} \frac{2 x_{3}-h}{h^{3}} \psi d y d x_{3}=\int_{B_{x}} q \frac{\partial}{\partial y_{1}}\left(\frac{x_{3}\left(x_{3}-h\right)}{h^{3}} \psi\right) d y d x_{3}$.

Integrating by parts in the second term on the left-hand side, and taking into account that $q$ and $\psi$ do not rely on $x_{3}$, we obtain by simple calculations

$$
\int_{B_{x}} l_{1} \frac{x_{3}}{h^{3}}\left(x_{3}-h\right) \psi d y d x_{3}-\int_{B_{x}} \frac{2}{h^{3}} l_{1} \psi d y d x_{3}=-\frac{1}{6} \int_{B_{x}} q \frac{\partial \psi}{\partial y_{1}} d y d x_{3},
$$

which implies

$$
\left\langle\frac{\partial q}{\partial y_{1}}, \psi\right\rangle=6 \int_{Y}\left(\int_{0}^{h} \frac{l_{1}}{h^{3}}\left[x_{3}\left(x_{3}-h\right)-2\right] d x_{3}\right) \psi d y .
$$

As $\int_{0}^{h} \frac{l_{1}}{h^{3}}\left[x_{3}\left(x_{3}-h\right)-2\right] d x_{3}$ belongs to $L^{2}(Y)$, we deduce that $\frac{\partial q}{\partial y_{1}}$ belongs to $L^{2}(Y)$.

Proceeding in the same manner in $y_{2}$, we prove that $q \in H^{1}(Y)$. So equality (4.20) is, in fact, in $L^{2}\left(B_{x}\right)$. Now, for any $\phi$ in $\left(H_{0 \#}^{1}\left(B_{x}\right)\right)^{3}$ with $\operatorname{div}_{B_{x}} \phi=0$, multiplying (4.20) by $\phi_{i}$ and using the Green formula, we obtain after addition

$$
\begin{aligned}
& \sum_{i=1}^{2} \int_{B_{x}}\left(l_{i} \phi_{i}+\frac{\partial l_{i}}{\partial x_{3}} \frac{\partial \phi_{i}}{\partial x_{3}}\right) d y_{1} d y_{2} d x_{3} \\
& \quad=\int_{B_{x}} q\left(\frac{\partial \phi_{1}}{\partial y_{1}}+\frac{\partial \phi_{2}}{\partial y_{2}}\right) d y_{1} d y_{2} d x_{3}-\int_{\partial B_{x}} q\left(\phi_{1} n_{1}+\phi_{2} n_{2}\right) d \sigma
\end{aligned}
$$

Since $\int_{B_{x}} q \frac{\partial \phi_{3}}{\partial y_{3}} d y d x_{3}=0$, we deduce that

$$
\int_{B_{x}} q\left(\frac{\partial \phi_{1}}{\partial y_{1}}+\frac{\partial \phi_{2}}{\partial y_{2}}\right) d y d x_{3}=0
$$

so comparing with (4.18), we obtain

$$
\int_{\partial B_{x}} q\left(\phi_{1} n_{1}+\phi_{2} n_{2}\right) d \sigma=0 \quad \forall \phi \in\left(H_{0 \#}^{1}\left(B_{x}\right)\right)^{3} \quad \text { with } \operatorname{div}_{B_{x}} \phi=0 .
$$

Set

$$
B_{0}=\left\{\left(y_{2}, x_{3}\right) \quad y_{2} \in\right] 0,1\left[\text { and } 0<x_{3}<h\left(x, 0, y_{2}\right)\right\} .
$$

For any $\psi$ in $\mathcal{D}\left(B_{0}\right)$, there is $\varphi \in\left(H_{0 \#}^{1}\left(B_{x}\right)\right)^{3}$ with $\operatorname{div}_{\lambda} \varphi=0$ such that (see [16])

$$
\begin{gathered}
\varphi / y_{1}=0=\varphi / y_{1}=1=(\psi, 0,0), \\
\varphi_{/ y_{2}=0}=\varphi_{/ y_{2}=1}=\varphi_{/ x_{3}=0}=\varphi_{/ x_{3}=h}=0 .
\end{gathered}
$$

We then deduce from (4.22) the $y_{1}$-periodicity of $q$, and in a similar way its $y_{2}$-periodicity, so $q \in H_{p e r}^{1}(Y)$. 
To end the proof of the lemma, we must show that $L$ defined in (4.17) is equal to zero for any $v$ in $V_{00}$. Multiplying (4.20) by $v_{i}$ and using the Green formula, we obtain

$$
\begin{aligned}
\sum_{i=1}^{2} \int_{B_{x}}\left(l_{i} v_{i}+\frac{\partial l_{i}}{\partial x_{3}} \frac{\partial v_{i}}{\partial x_{3}}\right) d y d x_{3} & =-\int_{B_{x}}\left(\frac{\partial q}{\partial y_{1}} v_{1}+\frac{\partial q}{\partial y_{2}} v_{2}\right) d y d x_{3} \\
& =-\int_{Y}\left(\int_{0}^{h} v d x_{3}\right) \nabla_{y} q d y
\end{aligned}
$$

since $q$ is independent on $x_{3}$. The last term is equal to zero as $v \in V_{00}$ and $q \in H_{p e r}^{1}(Y)$, which proves the result.

Proposition 4.9. There is $u$ in $\left(L^{2}\left(\Omega_{\max } ; H_{p e r}^{1}(Y)\right)\right)^{3}$ with $\frac{\partial u}{\partial x_{3}} \in\left(L^{2}\left(\Omega_{\max } \times Y\right)\right)^{3}$ and $v$ in $\left(L^{2}\left(\Omega_{\max } \times Y ; H_{p e r}^{1}([0,1])\right)^{3}\right.$ such that

$$
\begin{gathered}
\bar{u}^{\epsilon} \rightarrow u, \\
\frac{\partial \bar{u}^{\epsilon}}{\partial x_{3}} \rightarrow \frac{\partial u}{\partial x_{3}}+\frac{\partial v}{\partial y_{3}}, \\
\eta \frac{\partial \bar{u}_{i}^{\epsilon}}{\partial x_{3}} \rightarrow 0 .
\end{gathered}
$$

Moreover,

$$
\begin{gathered}
\int_{Y} \sum_{i=1}^{2}\left(\int_{0}^{h} u_{i}^{\lambda} d x_{3}\right) \frac{\partial \psi}{\partial y_{i}} d y=0 \quad \forall \psi \in H^{1}(Y) \\
u=0 \text { for } x_{3} \geq h(x, y) \text { and } u=\left(s_{1}, s_{2}, 0\right) \text { for } x_{3}=0 .
\end{gathered}
$$

Proof. The proof of all the results except (4.23) is similar to the ones in Proposition 4.6. Relation (4.23) is immediately deduced from $\operatorname{div}_{\eta} \bar{u}^{\epsilon}=0$ by taking a test function of the form $\theta(x) \psi(y)$ with $\theta \in \mathcal{D}(\omega)$ and $\psi \in H_{p e r}^{1}(Y)$.

Now we have to introduce the relation between the limit velocity and the gradient of the limit pressure. For that purpose, in addition to associated auxiliary problems, we will need a function $R$ in $\left(V_{x_{3}}\left(B_{x}\right)\right)^{3}$, which satisfies

$$
R_{/ x_{3}=0}=\left(s_{1}, s_{2}, 0\right) \text { and } R_{/ x_{3}=h}=0 .
$$

For example we take $R=\left(r_{1}, r_{2}, 0\right)$ where (for $\left.i=1,2\right)$

$$
r_{i}\left(x, x_{3}\right)=\left\{\begin{array}{lc}
s_{i}\left(1-\frac{x_{3}}{h_{\min }}\right) & \text { if } 0<x_{3}<h_{\min } \\
0 & x_{3} \geq h_{\min }
\end{array}\right.
$$

Proposition 4.10. The limit pressure $p$ belongs to $H^{1}(\omega)$ and the following relation is satisfied:

$$
u=w^{1} \frac{\partial p}{\partial x_{1}}+w^{2} \frac{\partial p}{\partial x_{1}}+w^{3}
$$

where $w^{k}(k=1,2,3)$ are the unique solutions of the auxiliary problems. 
Find $w^{k}(k=1,2)$ in $V_{00}$ such that

$$
\mu \sum_{i=1}^{2} \int_{B_{x}} \frac{\partial w_{i}^{k}}{\partial x_{3}} \frac{\partial \varphi_{i}}{\partial x_{3}} d y d x_{3}=-\int_{B_{x}} \varphi_{k} d y d x_{3} \quad \forall \varphi \in V_{00}
$$

and

Find $w^{3}$ in $R+V_{00}$ such that

$$
\mu \sum_{i=1}^{2} \int_{B_{x}} \frac{\partial w_{i}^{3}}{\partial x_{3}} \frac{\partial \varphi_{i}}{\partial x_{3}} d y d x_{3}=0 \quad \forall \varphi \in V_{00}
$$

with $R$ defined in (4.24).

Proof. Let $\psi=\left(\psi_{1}, \psi_{2}, \psi_{3}\right) \in\left(\mathcal{D}\left(\Omega_{\max }, C_{\#}^{\infty}(Y)\right)\right)^{3}$ with $\operatorname{div}_{B_{x}} \psi=0$ and $\psi=0$ for $x_{3} \geq h(x, y)$. Set $\psi^{\epsilon}\left(x, x_{3}\right)=\psi\left(x, \frac{x}{\epsilon}, x_{3}\right)$ and taking $\left(\psi_{1}^{\epsilon}, \psi_{2}^{\epsilon}, \frac{\eta}{\epsilon} \psi_{3}^{\epsilon}\right)$ as a test function in (2.7), we obtain

$$
\begin{array}{r}
\mu \int_{\Omega_{\max }}\left[\sum_{i, j=1}^{2} \frac{\partial \bar{u}_{i}^{\epsilon}}{\partial x_{j}} \cdot\left(\frac{\partial \psi_{i}}{\partial x_{j}}+\frac{1}{\epsilon} \frac{\partial \psi_{i}}{\partial y_{j}}\right)+\frac{\eta}{\epsilon} \frac{\partial \bar{u}_{3}^{\epsilon}}{\partial x_{3}} \cdot\left(\frac{\partial \psi_{3}}{\partial x_{j}}+\frac{1}{\epsilon} \frac{\partial \psi_{3}}{\partial y_{j}}\right)\right. \\
\left.+\frac{1}{\eta^{2}} \sum_{i=1}^{2} \frac{\partial \bar{u}_{i}^{\epsilon}}{\partial x_{3}} \cdot \frac{\partial \psi_{i}}{\partial x_{3}}+\frac{1}{\eta \epsilon} \frac{\partial \bar{u}_{3}^{\epsilon}}{\partial x_{3}} \cdot \frac{\partial \psi_{3}}{\partial x_{3}}\right] d x d x_{3}=\int_{\Omega_{\max }} p^{\epsilon}\left(\frac{\partial \psi_{1}}{\partial x_{1}}+\frac{\partial \psi_{2}}{\partial x_{2}}\right) d x d x_{3} .
\end{array}
$$

Passing to the limit after multiplication by $\eta^{2}$, we obtain

$$
\mu \int_{\omega \times B_{x}} \sum_{i=1}^{2} \frac{\partial u_{i}}{\partial x_{3}} \cdot \frac{\partial \psi_{i}}{\partial x_{3}} d x d y d x_{3}=\int_{\omega \times B_{x}} p\left(\frac{\partial \psi_{1}}{\partial x_{1}}+\frac{\partial \psi_{2}}{\partial x_{2}}\right) d x d x_{3} .
$$

Exactly as in the second step of the proof of Proposition 4.7. we prove that $p \in H^{1}(\omega)$ and that

$$
\text { a.e. } x \in \omega \quad \mu \sum_{i=1}^{2} \int_{B_{x}} \frac{\partial u_{i}}{\partial x_{3}} \cdot \frac{\partial \varphi_{i}}{\partial x_{3}} d x d y d x_{3}=-\sum_{i=1}^{2} \int_{B_{x}} \frac{\partial p}{\partial x_{i}} \varphi_{i}, d y d x_{3} \forall \varphi \in V .
$$

By the density Lemma 4.8, the above relation is true for any $\varphi \in V_{00}$.

From Proposition 4.9, $u-R$ belongs to $V_{00}$. On the other hand, by the Poincaré inequality, the semi-norm

$$
\left(\sum_{i=1}^{2} \int_{B_{x}}\left(\frac{\partial \varphi_{i}}{\partial x_{3}}\right)^{2} d y d x_{3}\right)^{\frac{1}{2}}
$$

is a norm in $V_{00}$. So from the Lax-Milgram theorem, $\left(u_{1}, u_{2}\right)$ is the unique solution in $R+V_{00}$ satisfying (4.30) for all $\varphi$ in $V_{00}$. Besides, for the same reasons, the auxiliary problems (4.26) and (4.27) have unique solutions that give (4.25) by linearity and the fact that $p$ depends on $x$ only.

LEMmA 4.11. The solutions to the auxiliary problems (4.26) and (4.27) satisfy

$$
w_{i}^{k}=\frac{1}{2 \mu}\left(\frac{\partial \pi^{k}}{\partial y_{i}}+\delta_{k i}\right) x_{3}\left(x_{3}-h\right)
$$


and

$$
w_{i}^{3}=\frac{1}{2 \mu} \frac{\partial \pi^{3}}{\partial y_{i}} x_{3}\left(x_{3}-h\right)+s_{i}\left(1-\frac{x_{3}}{h}\right)
$$

where $\pi^{k}(k=1,2,3)$ are the solutions of the local problems defined in (3.5) and (3.6).

Proof. As $V \subset V_{00}$, using the De Rham theorem in equation (4.26), there is $\widetilde{\pi}^{k}$ in $L_{0}^{2}\left(B_{x}\right)$ such that

$$
\begin{array}{r}
\mu \sum_{i=1}^{2} \int_{B_{x}} \frac{\partial w_{i}^{k}}{\partial x_{3}} \frac{\partial \varphi_{i}}{\partial x_{3}} d y_{1} d y_{2} d x_{3}=-\int_{B_{x}} \varphi_{k} d y_{1} d y_{2} d x_{3} \\
+\int_{B_{x}} \tilde{\pi}^{k} \operatorname{div}_{B_{x}} \varphi d y_{1} d y_{2} d x_{3} \quad \forall \varphi \in H_{0}^{1}\left(B_{x}\right)^{3}
\end{array}
$$

which implies, setting respectively $(\varphi, 0,0),(0, \varphi, 0)$ and $(0,0, \varphi)$ as test functions in (4.33), the following equations:

$$
\mu \frac{\partial^{2} w_{i}^{k}}{\partial x_{3}^{2}}=\delta_{i k}+\frac{\partial \widetilde{\pi}^{k}}{\partial y_{i}} \quad i, k=1,2
$$

and

$$
\frac{\partial \widetilde{\pi}^{k}}{\partial x_{3}}=0 \quad k=1,2 .
$$

In the same manner, as for $q$ in Lemma 4.10 , we prove that $\widetilde{\pi}^{k}$ are in $H_{\text {per }}^{1}(Y)$ so that (4.34) holds in $L^{2}\left(B_{x}\right)$.

Integrating twice in $x_{3}$, taking into account the boundary conditions and the fact that $\widetilde{\pi}^{k}$ does not rely on $x_{3}$, we obtain

$$
w_{i}^{k}=\frac{1}{2 \mu}\left(\frac{\partial \widetilde{\pi}^{k}}{\partial y_{i}}+\delta_{i k}\right) x_{3}\left(x_{3}-h\right) .
$$

Finally, $w^{k}$ belongs to $V_{00}$, so using (4.36), we obtain that $\widetilde{\pi}^{k}$ satisfy (3.5). By uniqueness $\tilde{\pi}^{k}=\pi^{k}$, which proves (4.31).

Proof of Theorem 3.2. Similarly to the beginning of the proof of Theorem 3.1, we obtain

$$
\int_{w} \int_{B_{x}}\left(u_{1} \frac{\partial \varphi}{\partial x_{1}}+u_{2} \frac{\partial \varphi}{\partial x_{2}}\right) d x d y d x_{3}=\int_{\partial w}\left(\int_{0}^{h_{m i n}} g d x_{3}\right) \cdot n \varphi d \sigma \quad \forall \varphi \in H^{1}(\omega) .
$$

Using (4.25), (4.31), and (4.32) in (4.37), we obtain the Reynolds equation (3.7).

We now prove that the matrix $A$, whose entries are given by (3.8), is symmetric and positive definite to prove the uniqueness of the solution in (3.7).

Set $\phi=\pi^{j}$ in (3.5):

$$
\int_{Y} \frac{h^{3}}{12 \mu}\left(e_{i}+\nabla_{y} \pi^{i}\right) \cdot \nabla_{y} \pi^{j} d y=0
$$

so, $a_{i j}$ can be written in the form

$$
a_{i j}(x)=\int_{Y} \frac{h^{3}}{12 \mu}\left(e_{i}+\nabla_{y} \pi^{i}\right) \cdot\left(e_{j}+\nabla_{y} \pi^{j}\right) d y
$$


which proves that $A(x)$ is symmetric.

For any $\xi=\left(\xi_{1}, \xi_{2}\right)$ in $\mathbb{R}^{2}$, we have

$$
\xi^{t} A \xi=\int_{Y} \frac{h^{3}}{12 \mu}\left(\sum_{i=1}^{2} \xi_{i}\left(e_{i}+\nabla_{y} \pi^{i}\right)\right)^{2} d y .
$$

Using (2.1),

$$
\begin{gathered}
\xi^{t} A \xi \geq \frac{a^{3}}{12 \mu} \int_{Y}\left\|\sum_{i=1}^{2} \xi_{i}\left(e_{i}+\nabla_{y} \pi^{i}\right)\right\|_{2}^{2} d y \\
=\frac{a^{3}}{12 \mu} \int_{Y}\left[\xi_{1}^{2}\left\|e_{1}+\nabla_{y} \pi^{1}\right\|_{2}^{2}+\xi_{2}^{2}\left\|e_{2}+\nabla_{y} \pi^{2}\right\|_{2}^{2}+2 \xi_{1} \xi_{2}\left(e_{1}+\nabla_{y} \pi^{1}\right) \cdot\left(e_{2}+\nabla_{y} \pi^{2}\right)\right] d y .
\end{gathered}
$$

On the other hand, for $i=1,2$,

$$
\int_{Y}\left\|\left(e_{i}+\nabla_{y} \pi^{i}\right)^{2}\right\|_{2}^{2} d y=\int_{Y}\left[1+\left\|\nabla_{y} \pi^{i}\right\|_{2}^{2}+2 \frac{\partial \pi^{i}}{\partial y_{i}}\right] d y=\int_{Y}\left[1+\left\|\nabla_{y} \pi^{i}\right\|_{2}^{2}\right] d y
$$

since $\pi^{i}$ is $y$-periodic. For the same reason, we have

$$
\int_{Y}\left(e_{1}+\nabla_{y} \pi^{1}\right) \cdot\left(e_{2}+\nabla_{y} \pi^{2}\right) d y=\int_{Y} \nabla_{y} \pi^{1} \cdot \nabla_{y} \pi^{2} d y .
$$

We then obtain

$$
\xi^{t} A \xi \geq \frac{a^{3}}{12 \mu} \int_{Y} \sum_{i=1}^{2} \xi_{i}^{2} d y+\frac{a^{3}}{12 \mu} \int_{Y}\left\|\xi_{1} \nabla_{y} \pi^{1}+\xi_{2} \nabla_{y} \pi^{2}\right\|_{2}^{2} d y \geq \frac{a^{3}}{12 \mu}\|\xi\|_{2}^{2} .
$$

The uniqueness of the solution of (3.7) is now assured.

\section{Proofs of theorems in Section 3.2.}

5.1. Proof of Theorem $3.3(\alpha=1)$. Let us introduce the modified stress matrix

$$
\mathcal{A}^{\epsilon}=\left(\begin{array}{ccc}
\eta^{2} h^{\epsilon} \sigma_{11}^{\epsilon} & \eta^{2} h^{\epsilon} \sigma_{12}^{\epsilon} & t_{3}\left(-\eta^{2} \sigma_{11}^{\epsilon} \frac{\partial h^{\epsilon}}{\partial t_{1}}-\eta^{2} \sigma_{12}^{\epsilon} \frac{\partial h^{\epsilon}}{\partial t_{2}}+\eta \sigma_{13}^{\epsilon}\right) \\
\eta^{2} h^{\epsilon} \sigma_{21}^{\epsilon} & \eta^{2} h^{\epsilon} \sigma_{22}^{\epsilon} & t_{3}\left(-\eta^{2} \sigma_{21}^{\epsilon} \frac{\partial h^{\epsilon}}{\partial t_{1}}-\eta^{2} \sigma_{22}^{\epsilon} \frac{\partial h^{\epsilon}}{\partial t_{2}}+\eta \sigma_{23}^{\epsilon}\right) \\
\eta 3 h^{\epsilon} \sigma_{31}^{\epsilon} & \eta 3 h^{\epsilon} \sigma_{22}^{\epsilon} & t_{3}\left(-\eta 3 \sigma_{31}^{\epsilon} \frac{\partial h^{\epsilon}}{\partial t_{1}}-\eta 3 \sigma_{32}^{\epsilon} \frac{\partial h^{\epsilon}}{\partial t_{2}}+\eta^{2} \sigma_{33}^{\epsilon}\right)
\end{array}\right)
$$

Note that the vector $V_{+}^{\epsilon}$ defined in (3.9) corresponds to the last column of the matrix $\mathcal{A}^{\epsilon}$ where $t_{3}=1$. So we first study the limit of the matrix $\mathcal{A}^{\epsilon}$ in order to deduce the limit of the vector $V_{+}^{\epsilon}$. For that purpose the following lemma is useful:

Lemma 5.1. The row vectors $\epsilon \mathcal{A}_{i}^{\epsilon}(i=1,2)$ and $\mathcal{A}_{3}^{\epsilon}$ of the matrix $\mathcal{A}^{\epsilon}$ are bounded in $H(\operatorname{div} ; \Omega)$. 
Proof. It is easy to prove that for $i=1,2,3$,

$$
\frac{\partial}{\partial t_{1}}\left(h^{\epsilon} \sigma_{i 1}^{\epsilon}\right)+\frac{\partial}{\partial t_{2}}\left(h^{\epsilon} \sigma_{i 2}^{\epsilon}\right)+\frac{\partial}{\partial t_{3}}\left(-t_{3} \frac{\partial h^{\epsilon}}{\partial t_{1}} \sigma_{i 1}^{\epsilon}-t_{3} \frac{\partial h^{\epsilon}}{\partial t_{2}} \sigma_{i 2}^{\epsilon}+\frac{1}{\eta} \sigma_{i 3}^{\epsilon}\right)=0
$$

so for $i=1,2$,

$$
\begin{aligned}
\operatorname{div}_{t t_{3}} \mathcal{A}_{i}^{\epsilon}=\eta^{2} h^{\epsilon} \sigma_{i 1}^{\epsilon} \frac{\partial}{\partial t_{1}} & \left(\frac{1}{\sqrt{1+\eta^{2}\left(\frac{\partial h^{\epsilon}}{\partial t_{1}}\right)^{2}+\eta^{2}\left(\frac{\partial h^{\epsilon}}{\partial t_{2}}\right)^{2}}}\right) \\
& +\eta^{2} h^{\epsilon} \sigma_{i 2}^{\eta \epsilon} \frac{\partial}{\partial t_{2}}\left(\frac{1}{\sqrt{1+\eta^{2}\left(\frac{\partial h^{\epsilon}}{\partial t_{1}}\right)^{2}+\eta^{2}\left(\frac{\partial h^{\epsilon}}{\partial t_{2}}\right)^{2}}}\right)
\end{aligned}
$$

and

$$
\begin{aligned}
\operatorname{div}_{t t_{3}} \mathcal{A}_{3}^{\epsilon}=\eta^{3} h^{\epsilon} \sigma_{31}^{\epsilon} \frac{\partial}{\partial t_{1}}( & \left.\frac{1}{\sqrt{1+\eta^{2}\left(\frac{\partial h^{\epsilon}}{\partial t_{1}}\right)^{2}+\eta^{2}\left(\frac{\partial h^{\epsilon}}{\partial t_{2}}\right)^{2}}}\right) \\
& +\eta^{3} h^{\epsilon} \sigma_{32}^{\eta \epsilon} \frac{\partial}{\partial t_{2}}\left(\frac{1}{\sqrt{1+\eta^{2}\left(\frac{\partial h^{\epsilon}}{\partial t_{1}}\right)^{2}+\eta^{2}\left(\frac{\partial h^{\epsilon}}{\partial t_{2}}\right)^{2}}}\right)
\end{aligned}
$$

Using the estimates of Lemma 4.2 on velocity and pressure and

$$
\left\|\frac{\partial h^{\epsilon}}{\partial t_{i}}\right\|_{L^{\infty}(w)}=\left\|\frac{\partial h}{\partial t_{i}}+\frac{1}{\epsilon} \frac{\partial h}{\partial y_{i}}\right\|_{L^{\infty}(w)} \leq \frac{K}{\epsilon}
$$

we deduce

$$
\eta^{2}\left\|\sigma_{i i}^{\epsilon}\right\|_{L^{2}(\Omega)} \leq K \quad \eta\left\|\sigma_{i j}^{\epsilon}\right\|_{L^{2}(\Omega)} \leq K \quad i \neq j=1,2,3 .
$$

The estimates (5.5) and (5.6) prove that

$$
\left\|\epsilon \mathcal{A}_{i}^{\epsilon}\right\|_{L 2(\Omega)^{3}} \leq K, \quad\left\|\epsilon \operatorname{div} \mathcal{A}_{i}^{\epsilon}\right\|_{L 2(\Omega)^{3}} \leq K \quad i=1,2
$$

and

$$
\left\|\mathcal{A}_{3}^{\epsilon}\right\|_{L 2(\Omega)^{3}} \leq K, \quad\left\|\operatorname{div} \mathcal{A}_{3}^{\epsilon}\right\|_{L 2(\Omega)^{3}} \leq K
$$

Now we prove the convergence of the stresses.

Proof of Theorem 3.3. According to the estimates of the previous lemma, there is a matrix $\mathcal{A}^{\lambda}$ in $L^{2}(\Omega)^{9}$ such that

$$
\epsilon \mathcal{A}_{i}^{\epsilon} \rightarrow \mathcal{A}_{i}^{\lambda} \quad i=1,2 ; \quad \text { and } \mathcal{A}_{3}^{\epsilon} \rightarrow \mathcal{A}_{3}^{\lambda} \quad \text { weakly in } H(\operatorname{div} ; \Omega) .
$$

We now identify the limit $\mathcal{A}^{\lambda}$. It is clear that the first two columns of $\mathcal{A}^{\lambda}$ are zero. For the third column, the only terms with a nonzero limit are

$$
\eta^{2} \epsilon t_{3} p^{\epsilon} \frac{\frac{\partial h^{\epsilon}}{\partial t_{i}}}{1+\eta^{2}\left(\frac{\partial h^{\epsilon}}{\partial t_{1}}\right)^{2} \eta^{2}\left(\frac{\partial h^{\epsilon}}{\partial t_{2}}\right)^{2}}, i=1,2
$$

and

$$
\eta^{2} \epsilon p^{\epsilon} \frac{\frac{\partial h^{\epsilon}}{\partial t_{i}}}{1+\eta^{2}\left(\frac{\partial h^{\epsilon}}{\partial t_{1}}\right)^{2} \eta^{2}\left(\frac{\partial h^{\epsilon}}{\partial t_{2}}\right)^{2}}, i=1,2
$$


We first remark that

$$
\frac{\epsilon \frac{\partial h^{\epsilon}}{\partial t_{i}}}{\sqrt{1+\eta^{2}\left(\frac{\partial h^{\epsilon}}{\partial t_{1}}\right)^{2}+\eta^{2}\left(\frac{\partial h^{\epsilon}}{\partial t_{2}}\right)^{2}}}=\frac{\frac{\partial h}{\partial y_{i}}}{\sqrt{1+\lambda^{2}\left(\frac{\partial h}{\partial y_{1}}\right)^{2}+\lambda^{2}\left(\frac{\partial h}{\partial y_{2}}\right)^{2}}}+e_{i}^{\epsilon}
$$

with

$$
\begin{aligned}
e_{i}^{\epsilon}= & \frac{\epsilon \frac{\partial h^{\epsilon}}{\partial t_{i}}-\frac{\partial h}{\partial y_{i}}}{\sqrt{1+\eta^{2}\left(\frac{\partial h^{\epsilon}}{\partial t_{1}}\right)^{2}+\eta^{2}\left(\frac{\partial h^{\epsilon}}{\partial t_{2}}\right)^{2}}} \\
& +\frac{\partial h}{\partial y_{i}}\left[\frac{1}{\sqrt{1+\eta^{2}\left(\frac{\partial h^{\epsilon}}{\partial t_{1}}\right)^{2}+\eta^{2}\left(\frac{\partial h^{\epsilon}}{\partial t_{2}}\right)^{2}}}-\frac{1}{\sqrt{1+\lambda^{2}\left(\frac{\partial h}{\partial y_{1}}\right)^{2}+\lambda^{2}\left(\frac{\partial h}{\partial y_{2}}\right)^{2}}}\right] .
\end{aligned}
$$

As $e_{i}^{\epsilon}$ strongly converges to zero in $L^{\infty}(\Omega)$, it is sufficient to find the weak limit of

$$
\eta^{2} p^{\epsilon} t_{3} \frac{\frac{\partial h}{\partial y_{i}}}{1+\lambda^{2}\left(\frac{\partial h}{\partial y_{1}}\right)^{2} \lambda^{2}\left(\frac{\partial h}{\partial y_{2}}\right)^{2}} .
$$

Now from the two-scale convergence of $\eta^{2} p^{\epsilon}$, we obtain

$$
\mathcal{A}^{\lambda}=\left(\begin{array}{ccc}
0 & 0 & \lambda^{2} t_{3}\left(\int_{Y} \frac{\frac{\partial h}{\partial y_{1}}}{\sqrt{1+\lambda^{2}\left(\frac{\partial h}{\partial y_{1}}\right)^{2}+\lambda^{2}\left(\frac{\partial h}{\partial y_{2}}\right)^{2}}} d y\right) p^{\lambda} \\
0 & 0 & \lambda^{2} t_{3}\left(\int_{Y} \frac{\frac{\partial h}{\partial y_{2}}}{\sqrt{1+\lambda^{2}\left(\frac{\partial h}{\partial y_{1}}\right)^{2}+\lambda^{2}\left(\frac{\partial h}{\partial y_{2}}\right)^{2}}} d y\right) p^{\lambda} \\
0 & 0 & \lambda^{2} t_{3}\left(\int_{Y} \frac{-1}{\sqrt{1+\lambda^{2}\left(\frac{\partial h}{\partial y_{1}}\right)^{2}+\lambda^{2}\left(\frac{\partial h}{\partial y_{2}}\right)^{2}}} d y\right) p^{\lambda}
\end{array}\right),
$$

which gives the result.

$$
\text { 5.2. Proof of Theorem } 3.4(\alpha>1) \text {. The divergence of } \mathcal{A}_{i}^{\epsilon} \text { involves terms like } \frac{\eta^{2}}{\epsilon^{3}} \text {, so }
$$

$$
\left\|\operatorname{div} \mathcal{A}_{i}^{\epsilon}\right\|_{L 2(\Omega)^{3}} \leq K \epsilon^{2 \alpha-3} \quad i=1,2 .
$$

These estimates cause three different cases to occur: $\alpha>\frac{3}{2}, \alpha=\frac{3}{2}, \alpha<\frac{3}{2}$. We also remark that

$$
\left\|\mathcal{A}_{3}^{\epsilon}\right\|_{L 2(\Omega)^{3}} \leq K,\left\|\operatorname{div} \mathcal{A}_{3}^{\epsilon}\right\|_{L 2(\Omega)^{3}} \leq K .
$$

Proof of Theorem 3.4. We begin by studying the convergence of $V_{+3}^{\epsilon}$. It is clear that there exists $\mathcal{A}_{3}^{\lambda}$ such that

$$
\mathcal{A}_{3}^{\epsilon} \rightarrow \mathcal{A}_{3}^{\lambda} \text { weakly in } H(\operatorname{div} ; \Omega) \text {. }
$$

It is easy to show that $\mathcal{A}_{3}^{\lambda}=(0,0,-p(x))$, which gives the result on $V_{+3}^{\epsilon}$ for $\alpha>1$.

We now study the convergences of $V_{+i}^{\epsilon}, i=1,2$. Since we cannot have a uniform estimate for $\left\|\mathcal{A}_{i}^{\epsilon}\right\|_{L^{2}(\Omega)}$, we define the trace on the upper part of the boundary as element of $H^{1}(\omega)$ in the following manner: 
Let $\varphi\left(t, t_{3}\right)=\theta(t) \psi\left(t_{3}\right)$, where $\theta \in H_{0}^{1}(\omega), \psi \in H^{1}(] 0,1[)$ with $\psi(0)=0, \psi(1)=1$ and $\int_{0}^{1} \psi d_{3}=1$. We define $V_{+i}^{\epsilon}$ by the duality

$$
\left\langle V_{+i}^{\epsilon}, \theta\right\rangle=\int_{\Omega} \operatorname{div} \mathcal{A}_{i}^{\epsilon} \varphi d t d x_{3}+\int_{\Omega} \mathcal{A}_{i}^{\epsilon} \nabla \varphi d t d x_{3} \text { for } i=1,2
$$

Exactly as in 22, this definition does not depend on the choice of $\psi$. We have

$$
\begin{array}{r}
\int_{\Omega} A_{i}^{\epsilon} \cdot \nabla \varphi d t d t_{3}=\sum_{j=1,2} \int_{\Omega} \eta^{2} \frac{h^{\epsilon}}{\sqrt{1+\eta^{2}\left(\frac{\partial h^{\epsilon}}{\partial t_{1}}\right)^{2}+\eta^{2}\left(\frac{\partial h^{\epsilon}}{\partial t_{2}}\right)^{2}}} \frac{\partial \theta}{\partial t_{j}} \sigma_{i j}^{\epsilon} \psi d t d t_{3} \\
+\int_{\Omega} \frac{1}{\sqrt{1+\eta^{2}\left(\frac{\partial h^{\epsilon}}{\partial t_{1}}\right)^{2}+\eta^{2}\left(\frac{\partial h^{\epsilon}}{\partial t_{2}}\right)^{2}}}\left(-t_{3} \eta^{2} \sigma_{i 1}^{\epsilon} \frac{\partial h^{\epsilon}}{\partial t_{1}}-t_{3} \eta^{2} \sigma_{i 2}^{\epsilon} \frac{\partial h^{\epsilon}}{\partial t_{2}}+\eta \sigma_{i 3}^{\epsilon}\right) \theta \frac{d \psi}{d t_{3}} d t d t_{3} .
\end{array}
$$

All integrals in the first term in (5.9) converge to zero because of the multiplication by $\eta^{2}$, except

$$
-\eta^{2} \int_{\Omega} \frac{h^{\epsilon}}{\sqrt{1+\eta^{2}\left(\frac{\partial h^{\epsilon}}{\partial t_{1}}\right)^{2}+\eta^{2}\left(\frac{\partial h^{\epsilon}}{\partial t_{2}}\right)^{2}}} p^{\epsilon} \frac{\partial \theta}{\partial t_{i}} \psi d t d t_{3}
$$

Using the two-scale convergence of $\eta^{2} p^{\epsilon}$ to $p$, we easily deduce that

$$
\begin{array}{r}
\lim _{\epsilon \rightarrow 0}\left(-\eta^{2} \int_{\Omega} \frac{h^{\epsilon}}{\sqrt{1+\eta^{2}\left(\frac{\partial h^{\epsilon}}{\partial t_{1}}\right)^{2}+\eta^{2}\left(\frac{\partial h^{\epsilon}}{\partial t_{2}}\right)^{2}}} p^{\epsilon} \frac{\partial \theta}{\partial t_{i}} \psi d t d t_{3}\right) \\
=-\int_{\Omega} \int_{Y} h p \frac{\partial \theta}{\partial t_{i}} \psi d t d y d t_{3}=-\int_{\omega} \tilde{h} p \frac{\partial \theta}{\partial t_{i}} d t
\end{array}
$$

the last equality being a consequence of the hypothesis $\int_{0}^{1} \psi d t_{3}=1$.

In the second term in (5.9), all integrals converge to zero, except

$$
\int_{\Omega} \eta^{2} p^{\epsilon} t_{3} \frac{\frac{\partial h^{\epsilon}}{\partial t_{i}}}{\sqrt{1+\eta^{2}\left(\frac{\partial h^{\epsilon}}{\partial t_{1}}\right)^{2}+\eta^{2}\left(\frac{\partial h^{\epsilon}}{\partial t_{2}}\right)^{2}}} \theta \frac{d \psi}{d t_{3}} d t d t_{3} \quad i=1,2
$$

and

$$
\mu \int_{\Omega} \frac{1}{h^{\epsilon}} \frac{1}{\sqrt{1+\eta^{2}\left(\frac{\partial h^{\epsilon}}{\partial t_{1}}\right)^{2}+\eta^{2}\left(\frac{\partial h^{\epsilon}}{\partial t_{2}}\right)^{2}}} \frac{\partial u_{i}^{\epsilon}}{\partial t_{3}} \theta \frac{d \psi}{d t_{3}} \quad d t d t_{3} \quad i=1,2 .
$$

We now prove that the term in (5.11) tends to zero. We set $\xi\left(t_{3}\right)=\int_{0}^{t_{3}} s \frac{d \psi}{d s} d s$ $=t_{3} \psi\left(t_{3}\right)-\int_{0}^{t_{3}} \psi(s) d s, \xi(0)=\xi(1)=0$ and $\frac{d \xi}{d t_{3}}=t_{3} \frac{d \psi}{d t_{3}}$. Choosing $\varphi=\left(0,0, \varphi_{3}\left(t, t_{3}\right)\right)$ 


$$
\begin{gathered}
\text { with } \varphi_{3}\left(t, t_{3}\right)=\xi\left(t_{3}\right) \theta(t) \frac{\frac{\partial h^{\epsilon}}{\partial t_{i}}}{\sqrt{1+\eta^{2}\left(\frac{\partial h^{\epsilon}}{\partial t_{1}}\right)^{2}+\eta^{2}\left(\frac{\partial h^{\epsilon}}{\partial t_{2}}\right)^{2}}} \text { as a test function in (2.10) } \text {, we obtain } \\
\qquad \begin{array}{c}
\mu \int_{\Omega}\left[\sum_{i=1}^{2}\left(\frac{\partial}{\partial t_{i}}\left(\eta h^{\epsilon} u_{3}^{\epsilon}\right)+\frac{\partial}{\partial t_{3}}\left(-\eta t_{3} \frac{\partial h^{\epsilon}}{\partial t_{i}} u_{3}^{\epsilon}\right)\right) \cdot\left(\frac{\partial}{\partial t_{i}}\left(\eta h^{\epsilon} \varphi_{3}\right)+\frac{\partial}{\partial t_{3}}\left(-\eta t_{3} \frac{\partial h^{\epsilon}}{\partial t_{i}} \varphi_{3}\right)\right)\right. \\
\left.+\frac{\partial u_{3}^{\epsilon}}{\partial t_{3}} \frac{\partial \varphi_{3}}{\partial t_{3}}\right] \frac{d t d t_{3}}{\eta h^{\epsilon}}=\int_{\Omega} p^{\epsilon} \frac{\frac{\partial h^{\epsilon}}{\partial t_{i}}}{\sqrt{1+\eta^{2}\left(\frac{\partial h^{\epsilon}}{\partial t_{1}}\right)^{2}+\eta\left(\frac{\partial h^{\epsilon}}{\partial t_{2}}\right)^{2}}} \theta(t) t_{3} \frac{d \psi}{d t_{3}} d t d t_{3} .
\end{array}
\end{gathered}
$$

Using estimates on the velocity and (5.5), we deduce that the term in (5.11) tends to zero.

In order to pass to the limit in (5.12), we recall that

$$
\frac{\partial u_{i}^{\epsilon}}{\partial x_{3}} \rightarrow \frac{\partial u_{i}}{\partial x_{3}}+\frac{\partial v_{i}}{\partial y_{3}} \text { in } \Omega,
$$

so passing in $\Omega_{\max }$, we obtain

$$
\begin{array}{r}
\mu \int_{\Omega} \frac{1}{h^{\epsilon}} \frac{1}{\sqrt{1+\eta^{2}\left(\frac{\partial h^{\epsilon}}{\partial t_{1}}\right)^{2}+\eta^{2}\left(\frac{\partial h^{\epsilon}}{\partial t_{2}}\right)^{2}}} \frac{\partial u_{i}^{\epsilon}}{\partial t_{3}} \theta \frac{d \psi}{d t_{3}} d t d t_{3} \\
=\mu \int_{\Omega_{\max }} \frac{\partial u_{i}^{\epsilon}}{\sqrt{1+\eta^{2}\left(\frac{\partial h^{\epsilon}}{\partial x_{1}}\right)^{2}+\eta^{2}\left(\frac{\partial h^{\epsilon}}{\partial x_{2}}\right)^{2}}} \theta \psi^{\prime}\left(\frac{x_{3}}{h^{\epsilon}}\right) \frac{1}{h^{\epsilon}} d x d x_{3} .
\end{array}
$$

The limit of this term is

$$
\mu \int_{\Omega_{\max }} \int_{Y} \frac{\partial u_{i}}{\partial x_{3}} \theta \psi^{\prime}\left(\frac{x_{3}}{h}\right) \frac{1}{h} d x d x_{3} d y=\mu \int_{\Omega} \int_{Y} \frac{\partial u_{i}}{\partial t_{3}} \theta \psi^{\prime}\left(t_{3}\right) \frac{1}{h} d t d t_{3} d y .
$$

Using the Green formula and the fact that $\psi(1)=1$, we can write the last term as

$$
-\mu \int_{\Omega} \int_{Y} \frac{\partial^{2} u_{i}}{\partial t_{3}^{2}} \theta \psi \frac{1}{h} d t d t_{3} d y+\mu \int_{\omega} \int_{Y} \frac{\partial u_{i}}{\partial t_{3}}(t, y, 1) \theta \frac{1}{h} d t d y .
$$

On the other hand, from (4.25) and Lemma 4.11, we deduce

$$
\frac{\partial u_{i}}{\partial t_{3}}=\sum_{j=1}^{2} \frac{1}{2 \mu} \frac{\partial p}{\partial t_{j}}\left(\frac{\partial \pi^{j}}{\partial y_{i}}+\delta_{i j}\right) h^{2}\left(2 t_{3}-1\right)+\frac{1}{2 \mu} \frac{\partial \pi^{3}}{\partial y_{i}} h^{2}\left(2 t_{3}-1\right)-s_{i}
$$

and

$$
\mu \frac{\partial^{2} u_{i}}{\partial t_{3}^{2}}=\sum_{k=1}^{2} \frac{\partial p}{\partial t_{i}} h^{2}\left(\frac{\partial \pi^{k}}{\partial y_{i}}+\delta_{k i}\right)+h^{2} \frac{\partial \pi^{3}}{\partial y_{i}} .
$$

We then deduce that the limit of (5.12) is

$$
-\int_{\omega} \sum_{j=1}^{2} \frac{\partial p}{\partial t j} c_{i j}(t) \theta d t-\int_{\omega} d_{i} \theta d t
$$

and with the help of (5.9) (5.10), we deduce

$$
\lim _{\epsilon \rightarrow 0}\left(\int_{\Omega} \mathcal{A}_{i}^{\epsilon} \cdot \nabla \varphi d t d t_{3}\right)=\left\langle\frac{\partial}{\partial t_{i}}(p \widetilde{h})-\sum_{j=1}^{2} \frac{\partial p}{\partial t_{j}} c_{i j}-d_{i}, \theta\right\rangle_{H^{-1}(\omega), H_{0}^{1}(\omega)} .
$$


We now seek the limit of the term with $\operatorname{div} \mathcal{A}_{\mathrm{i}}^{\epsilon}$ in (5.8). We must consider three different cases on $\alpha$ :

i) For $\alpha>\frac{3}{2}$, (5.7) induces that $\int_{\Omega} \operatorname{div} \mathcal{A}_{i}^{\epsilon} \varphi d t d x_{3} \rightarrow 0$, which gives the desired convergence of Theorem 3.4 for $i=1,2$.

ii) For $\alpha=\frac{3}{2}$, using (5.3) and the two-scale convergence of $\eta^{2} p^{\epsilon}$, we obtain

$$
\lim _{\epsilon \rightarrow 0} \int_{\Omega} \operatorname{div} \mathcal{A}_{i}^{\epsilon} \theta \psi d t d t_{3}=\int_{\Omega} \int_{Y} \lambda^{2} p h \frac{\partial h}{\partial y_{i}} \frac{\partial^{2} h}{\partial y_{i}^{2}} \theta \psi d t d y d t_{3}=-\frac{\lambda^{2}}{2} \int_{\omega} p \widetilde{\left(\frac{\partial h}{\partial y_{i}}\right)^{3}} \theta d t
$$

which gives the result for $i=1,2$.

iii) For $1<\alpha<\frac{3}{2}$, we obtain the result multiplying (5.8) by $\epsilon^{3-2 \alpha}$, since

$$
\lim _{\epsilon \rightarrow 0}\left(\epsilon^{3-2 \alpha} \int_{\Omega} \mathcal{A}_{i}^{\epsilon} \cdot \nabla \varphi d t d t_{3}\right)=0
$$

and

$$
\lim _{\epsilon \rightarrow 0}\left(\epsilon^{3-2 \alpha} \int_{\Omega} \operatorname{div} \mathcal{A}_{i}^{\epsilon} \theta \psi d t d t_{3}\right)=-\int_{\omega} \frac{\lambda^{2}}{2} p \widetilde{\left(\frac{\partial h}{\partial y_{i}}\right)^{3}} \theta d t
$$

\section{REFERENCES}

[1] Y. Achdou, O. Pironneau, F. Valentin, Effective boundary conditions for laminar flows over periodic rough boundaries, J. Comput. Phys. 147 (1998), no. 1, 187-218. MR.1657773 (99j:76086)

[2] A. Ait Moussa, C. Licht, P. Suquet, Modélisation et singularités de contraintes d'un joint collé mince, Actes $9^{e}$ Congrès français de Mécanique (Metz) (1989), 258-259.

[3] G. Allaire, Homogenization and two-scale convergence, SIAM J. Math. Anal. 23 (1992), 1482-1518. MR:1185639 (93k:35022)

[4] Y. Amirat, D. Bresch, J. Lemoine, J. Simon, Effect of rugosity on a flow governed by stationary Navier-Stokes equations, Quart. Appl. Math. 59 (2001), no. 4, 769-785. MR1866556(2002g:76036)

[5] G. Bayada, M. Chambat, Homogenization of the Stokes system in a thin film flow with rapidly varying thickness, RAIRO Modél. Math. Anal. Numer. 23 (1989), no. 2, 205-234. MR1001328 (90e:76060)

[6] G. Bayada, M. Chambat, New models in the theory of the hydrodynamic lubrication of rough surfaces, Trans. of the AMS., J. of Trib. 110 (1988), 402-407.

[7] G. Bayada, M. Chambat and J. B. Faure, Some effects of the boundary roughness in a thin film flow, Preprint no. 58 1986, Lyon1. MR0942450 (89i:76044)

[8] G. Bayada, M. Chambat, K. Lhalouani, Asymptotic analysis of a thin-layer device with Tresca's contact law in elasticity, Math. Method Appl. Sci. 22 (1999), no. 10, 811-836. MR1700180 (2000f:74056)

[9] H. Brézis, Analyse fonctionnelle, Théorie et applications, Dunod, Paris, 1999. MR0697382 (85a:46001)

[10] I. Charpentier, J. Saint Jean Paulin, Limit behaviour of a three-dimensional thin tall structure depending on three small parameters, Ricerche. Mat. 44 (1995), no. 2, 459-488. MR1469715 (98i:73003)

[11] H. Christensen and K. Tonder, The hydrodynamic lubrication of rough bearing surfaces of finite width, J. of Lub. Tech., Trans. ASME, F 93 (1971), no. 3, 324-330.

[12] M. Dauge, Stationary Stokes and Navier-Stokes systems on two- or three-dimensional domains with corners, Part 1 - linearized equations, SIAM J. Math. Anal. 20 (1989) 74-97. MR0977489 (90b:35191)

[13] A. Dyson, Hydrodynamic lubrication of rough surfaces a review work, Proceedings of the 4th LeedsLyon Symposium on surfaces roughness on lubrication, 1977, IME, 61-69. 
[14] H. G. Elrod, A review of theories for the fluid dynamic effects of roughness on laminar lubricating films, Proceedings of the 4th Leeds-Lyon Symposium on surfaces roughness on lubrication, 1977, IME, 11-26.

[15] J. B. Faure, Application des techniques d'homogénéisation à la prise en compte des phénomènes de rugosité en lubrification hydrodynamique, thèse, Lyon 1, 1986.

[16] V. Girault and P.-A. Raviart, Finite Element Approximation of the Navier-Stokes Equation, Springer-Verlag, 1979. MR0548867 (83b:65122)

[17] W. Jäger, A. Mikelić, Couette flows over a rough boundary and drag reduction, Comm. Math. Phys. 232 (2003), no. 3, 429-455. MR.1952473 (2003j:76025)

[18] C. Licht, Comportement asymptotique d'une bande dissipative mince de faible rigidité, C. R. Acad. Sci. (Paris) 322, 429-433. MR1235462 (95b:73025)

[19] G. Nguetseng, A general Convergence Result for a Functional related to the Theory of Homogenization, SIAM J. Math. Anal. 20 (1989), no. 3, 608-623. MR0990867|(90j:35030)

[20] N. Phan Thien, Hydrodynamic lubrication of rough surfaces, Proc. R. Soc. London, A383 (1982) 439-446.

[21] E. Sanchez-Palencia, Non homogeneous media and vibration theory, Lecture Notes in Physics 127, Springer Verlag, Berlin, 1978.

[22] R. Temam, Navier-Stokes Equations, North Holland, Amsterdam, 1979. MR0603444 (82b:35133)

[23] R. Temam, Autour de la Mécanique des fluides, Cours de Dea, ENS de Lyon, Mars 2001. 